\title{
Compound Analysis of Jing Liqueur and nrf2 Activation by Jing Liqueur-One of the Most Popular Beverages in China
}

\author{
You-Sheng Cai ${ }^{1,2}$, Jian $\mathrm{Xu}^{3}{ }^{3}$, Mosi Chen ${ }^{3}$, Daoqing Wang ${ }^{3}$, Yuejun Yang ${ }^{3}$, \\ Arulmani Manavalan ${ }^{1}$, Xiaohua $\mathrm{Wu}^{1}$, Yuancai Liu ${ }^{3, *}$ and Shugeng Cao ${ }^{1, *}$ (D) \\ 1 Department of Pharmaceutical Sciences, Daniel K Inouye College of Pharmacy, University of Hawaii at Hilo, \\ 200 W. Kawili Street, Hilo, HI 96720, USA; caiyoush@hawaii.edu (Y.-S.C.); arulmani@hawaii.edu (A.M.); \\ xiaohua3@hawaii.edu (X.W.) \\ 2 Institute of TCM and Natural Products, School of Pharmaceutical Sciences, Wuhan University, \\ 185 Donghu Road, Wuhan 430071, China \\ 3 Jing Brand Research Institute, Jing Brand Co., Ltd., Daye 435100, China; xujian@jingpai.com (J.X.); \\ ccmmss@sina.com (M.C.); jt1269@126.com (D.W.); yyj@jingpai.com (Y.Y.) \\ * Correspondence: lyc@jingpai.com (Y.L.); scao@hawaii.edu (S.C.); Tel.: +86-71-4876-8056 (Y.L.); \\ $+1-808-981-8010$ (S.C.)
}

Received: 19 November 2019; Accepted: 24 December 2019; Published: 31 December 2019

\begin{abstract}
The aim of this study is to identify the minor compounds in Jing liqueur, determine the concentration of metals, amino acids, and polysaccharides, and evaluate their Nrf2 activity and cytotoxicity. Jing liqueur that contains Chinese medicine is one of the best-selling liqueurs in China, which is also marketed in the United States. Totally, we have isolated 189 minor compounds including one new molecule (7) from a concentrated Jing liqueur, with the concentrations of most isolated compounds at micromolar levels. The structures of all these compounds were determined by using MS and NMR (1D and 2D) or by comparison of their chemical and physical data with reported values in the literatures. Besides, the concentrations of iron $(0.52 \mathrm{mg} / \mathrm{L})$, zinc $(0.21 \mathrm{mg} / \mathrm{L})$, calcium $(11.0 \mathrm{mg} / \mathrm{L})$, $L$-proline $(2.33 \mathrm{mg} / \mathrm{L}), L$-arginine $(1.73 \mathrm{mg} / \mathrm{L})$, total amino acids $(9.84 \mathrm{mg} / \mathrm{L})$, and total polysaccharides $(337.4 \mathrm{mg} / \mathrm{L})$ were determined. Jing liqueur, the five fractions and most of the compounds isolated from Jing liqueur were screened for their activities in the Nrf2-ARE and MTT assays. At $5.2 \mathrm{mg} / \mathrm{mL}$ the crude enhanced the Nrf2 activity. At $80 \mu \mathrm{g} / \mathrm{mL}$, fraction IV weakly but fraction V strongly activated Nrf2. Among the compounds screened in the Nrf2 assay, eighteen activated Nrf2 at $40 \mu \mathrm{g} / \mathrm{mL}$ and compounds 51 and $\mathbf{1 2 6}$ from fraction $\mathrm{V}$ were the most active. The crude, all the five fractions, and Nrf2 activators were not cytotoxic toward HepG2 cells. In conclusion, Jing liqueur contains different classes of compounds including flavonoids, terpenoids, alkaloids, coumarins, cinnamic acid or coumaric acid, and phenyl ethanol (or acetic acid) derivatives, benzoquinone, naphthoquinone, anthraquinones or phenanphrene derivatives, xanthones, chromone, and $\gamma$-pyrone derivatives, lignans, other aromatic compounds, and others. Jing liqueur and the eighteen compounds, which were isolated from Jing liqueur, could activate Nrf2 without any cytotoxicity.
\end{abstract}

Keywords: Jing liqueur; traditional Chinese medicine; compounds; NMR; MS; Nrf2-ARE

\section{Introduction}

Jing liqueur [1-3] is a popular health beverage in China, which contains biologically active components from several tonic traditional Chinese herbal medicines. About 30 years ago, Jing Brand Co., Ltd. (Daye City, Hubei Province, China) began to sell its products overseas. Nowadays, Jing liqueur is sold in more than 20 countries and districts, including Hong Kong, Macau, Japan, South 
Korea, Australia, and the United States etc. The liqueur is manufactured using modern bioengineering technology to prepare extracts from Chinese herbal medicines such as Astragalus membranaceus, Cistanche deserticola, Dioscorea opposita, Lycium barbarum, Epimedium brevicornum, Cinnamomum cassia, Syzygium aromaticum, Angelica sinensis, and Imperata cylindrica, several of which are also used as foods or dietary supplements. These Chinese herbal medicines are carefully selected and prepared according to their applications.

A. membranaceus is a very common traditional Chinese medicine (TCM) widely used as an immunostimulant, cardiotonic, hepatoprotective, antidiabetic, antitumor, and antiviral drug [4]. C. deserticola is a famous Chinese Materia Medica (CMM) used for the treatment of kidney deficiency, infertility, and chronic constipation [5]. D. opposita is a famous tonic Chinese medicine with beneficial effects on spleen, lung, and kidney in addition to the antidiarrheal activity. The tuber is also a favorite food in China used in a stir-fry or in soups [6]. L. barbarum a traditional food and medicine in East Asia has become increasingly popular in Europe and North America in recent years. L. barbarum is used in folk medicine to increase longevity and is reported to have beneficial effects on blurry vision and diminished visual acuity, infertility, abdominal pain, dry cough, fatigue, and headache [6]. L. barbarum is also a very popular ingredient in Chinese cuisine, which is consumed in soups, as porridge with rice, and added to numerous meat and vegetable dishes [7]. E. brevicornum is one of the most commonly used traditional Chinese medicines, and has the reported benefits of reinforcing the "kidney yang," strengthening the tendons and bones, and relieving rheumatic conditions. It is also used to treat impotence, seminal emission, weakness of the limbs, rheumatoid arthralgia, and hypertension [8]. C. cassia is used in traditional Chinese medicine for various ailments including abdominal pain, vomiting, diarrhea, dysmenorrhea, blood stasis, bruises, and traumatic bleeding. It is also used as an appetite stimulant and a flavoring agent [9]. S. aromaticum is a Chinese medicine that is used as an aromatic stomachic agent, to relieve abdominal bloating, increase gastric secretions, aid in digestion, and reduce nausea and vomiting. It is also one of the most ancient and valuable spices of the Orient [10]. A. sinensis is one of the most important drugs in traditional Chinese medicine, and is commonly used for treating gynecopathias, including anemia, dysmenorrhea, amenorrhea, premenstrual, and menopausal syndromes. It is also used in the management of cancer, cardiovascular diseases, and Alzheimer's disease. Chicken soup made with Radix Angelica is a popular dish in China [11]. I. cylindrica is also a traditional Chinese medicine used in treating hot blood, blood vomiting, blood stasis, hematuria, fever, polydipsia, damp heat jaundice, edema, reduced urine output, and painful urination [12].

Nuclear factor erythroid 2-related factor 2 (Nrf2) is a transcription factor that controls the expression of antioxidant and phase II detoxifying enzymes. Nrf2 is widely recognized for its cytoprotective role, and has defensive properties against neurodegenerative, airway, and cardiac diseases [13]. Nrf2 is also targeted for the prevention of cancer and other chronic diseases such as diabetes, where oxidative stress and inflammation contribute to pathogenesis [14]. Also, Nrf2 activation through cell lineage-specific Keap1 disruption is important for the improvement of autoimmune diseases [15]. In these settings, transient activation of Nrf2 by compounds such as sulforaphane or curcumin can stimulate the expression of Nrf2 target genes to combat oxidative and electrophilic stress, reorganize cortical actin, reduce stress fibers formation, and improve the integrity of cell-cell junctions [16]. On one hand, Nrf2 activators could be used for the prevention of chemical carcinogenesis, whereas Nrf2 inhibitors could be used for cancer treatment [17].

Although the phytochemical composition and pharmacological properties of these nine Chinese herbal medicines have been independently evaluated, Jing liqueur containing extracts from these nine herbal medicines has not been previously investigated much. It has been reported that Jing liqueur showed anti-inflammatory [1], immune enhancement [3], anti-fatigue [2,3] properties, and enhancing Shen-Yang (kidney Yang) or invigorating the vital activities of kidney [3]. We argue that the anti-inflammation and immune enhancement of Jing liqueur may be due to or at least partially due to the Nrf2 activation by these traditional Chinese herbal medicines used in Jing liqueur. Hence, we also 
decided to evaluate Jing liqueur for its effect on Nrf2 besides the analysis of minor compounds. In this study, we isolated one hundred eighty nine (189) minor compounds from Jing liqueur including a new flavonoid (7), and determined their structures based on the MS data and NMR spectra. In addition, we determined the concentrations of iron, zinc, calcium, L-proline, L-arginine, total amino acids, and total polysaccharides. We also evaluated the effects of the crude Jing liqueur, the five fractions, and majority of the isolated compounds on the Nrf2 activity in a cell-based assay, and investigated the cytotoxicity of the crude Jing liqueur, the five fractions and the identified Nrf2 activators in a MTT assay. At $40 \mu \mathrm{g} / \mathrm{mL}$, eighteen compounds demonstrated Nrf2 activation without any cytotoxicity, and compound 51 was slightly less active while compound $\mathbf{1 2 6}$ was more active than the positive control SF $(5 \mu \mathrm{M})$, indicating that compounds $\mathbf{5 1}$ and $\mathbf{1 2 6}$ might be responsible for or partially account for the Nrf2 activation.

\section{Materials and Methods}

\subsection{Plant Materials}

Plant materials were collected in 2017 by researchers at Jing Brand Research Institute. Voucher specimens (JP20170219, A. membranaceus, Min County, Dingxi City, Gansu Province, China; JP20170259, C. deserticola, Hetian City, Xinjiang Uygur Autonomous Region, China; JP20170014, D. opposita, Jiaozuo City, Henan Province, China; JP20170214, L. barbarum, Zhongning County, Ningxia Hui Autonomous Region, China; JP20170244, E. brevicornum, Min County, Dingxi City, Gansu Province, China; JP20170143, C. cassia, Fangchenggang City, Guangxi Zhuang Autonomous Region, China; JP20170265, S. aromaticum, Indonesia; JP20170133, A. sinensis, Min County, Dingxi City, Gansu Province, China; and JP20170000, I. cylindrica, Louzhou City, Sichuan Province, China) are deposited at the herbarium of Jing Brand Research Institute, Daye City, Hubei Province, People's Republic of China.

\subsection{Preparation of Jing Liqueur}

Jing liqueur was prepared at Jing Brand Co., Ltd., using the company's proprietary technology. Following is a basic description of the process: (a). The raw Chinese herbal medicine (Astragalus membranaceus, Cistanche deserticola, Dioscorea opposita, Lycium barbarum, Epimedium brevicornum, Cinnamomum cassia, Syzygium aromaticum, Angelica sinensis, and Imperata cylindrica) was washed, dried, and sliced into pieces according to the protocols as described in the Chinese Pharmacopoeia. (b). Pieces of each herbal medicine were added to the "Xiaoqu white liqueur" with an alcohol content of $35 \%$ according to the company's process recipe. After percolation, filtration, and evaporation, various concentrated mother liquids were obtained. (c). The concentrated mother liquids were added to the "Xiaoqu white liqueur" with an alcohol content of 35\% for precise blending according to a standardized process recipe. (d). Certain amount of white sugar was added to adjust the taste. (e). The finished product was kept in a storage tank and stored for one year. After quality control, Jing liqueur was filled into small bottles and packaged for shipping to commission merchants.

\subsection{Concentration of Jing Liqueur}

Before white sugar was added, one hundred sixty liters (160 L) of Jing Brand "Xiaoqu white liqueur" (a semi-finished product after step c in the Section 2.2) was concentrated under vacuum to yield a syrup-like liquid, which was about $232 \mathrm{~g}$ if completely dried and was used for the separation and purification of minor compounds.

\subsection{HP20 Open Column, Preparative and Semi-Preparative HPLC}

To generate five fractions for the Nrf2 and cytotoxicity assay, $20 \mathrm{~mL}$ Jing liqueur was dried to yield a sample $(1.66 \mathrm{~g})$ in a pilot study. The sample was dissolved in $10 \mathrm{~mL}$ water, and loaded onto an open column (HP20 $6.6 \mathrm{~g}, 1.5 \times 6.0 \mathrm{~cm}$ ). HP20 is based on a unique rigid polystyrene/divinylbenzene matrix, in which a controlled pore size distribution and large surface area offer excellent resolution 
and the capacity for a wide range of molecules. The separation mechanism of a HP20 column is very similar to that of the C18 reverse chromatography-the most polar compounds are eluted out of the column with water first, while the most non-polar compounds will be eluted out of the column with methanol. Hence, a gradient solvent system from $100 \%$ water to $100 \%$ methanol $(0,20,50,80$, and $100 \% \mathrm{MeOH} / \mathrm{H}_{2} \mathrm{O}$ ) was used for the HP20 open column separation, and the eluents were dried using SpeedVac to yield five fractions (Fr. I: 1.5 g; Fr. II: 134 mg; Fr. III: 93.0 mg; Fr. IV: 8.0 mg; Fr. V: 1.3 $\mathrm{mg}$ ). Separation of large amount of $160 \mathrm{~L}$ Jing Brand "Xiaoqu white liqueur" sample was scaled up accordingly. Fraction I was mainly composed of saccharides, which was not chemically investigated in this study. Fractions II, III, IV, and V each were first separated with a Thermo Scientific Ultimate 3000 preparative high performance liquid chromatography (HPLC) system (Column: Phenomenex Luna C18, $100 \AA, 100 \times 21.2 \mathrm{~mm}, 5 \mu \mathrm{m}$; Flow-rate: $10 \mathrm{~mL} / \mathrm{min}$ ) and then an Agilent 1100 semi-preparative HPLC system (Column: Phenomenex Luna C18 or C8, $100 \AA$ A, $250 \times 10$ mm, $5 \mu \mathrm{m}$; Flow-rate: $3 \mathrm{~mL} / \mathrm{min}$ ) to get pure compounds (Scheme 1).

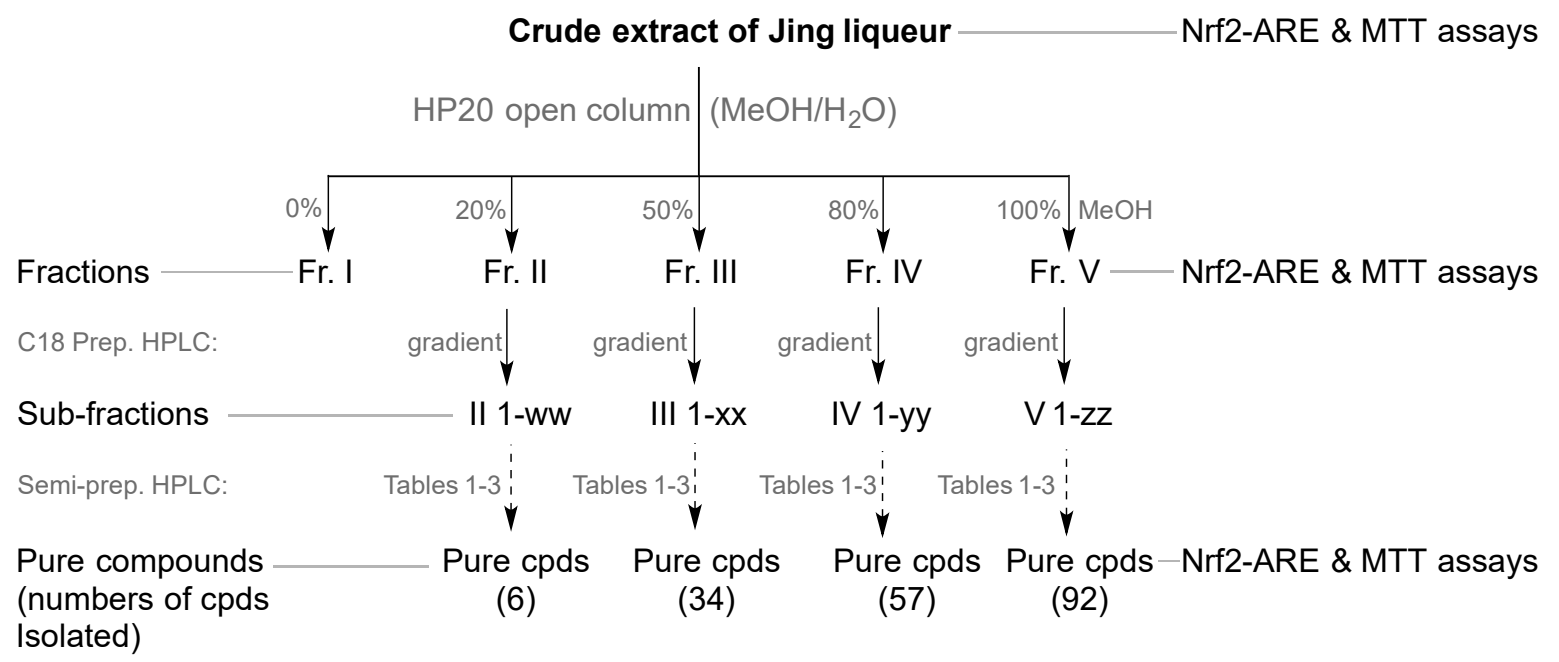

Scheme 1. Flow chart of experimental design and numbers of pure compounds isolated from fractions II-V (See Tables 1-3 for retention times of the 189 pure compounds and high performance liquid chromatography (HPLC) conditions including columns, flow-rates, and solvent systems).

\subsection{LC/MS Condition for the Analysis}

System: Agilent 1260 HPLC coupled to 6120 quadrupole LC/MS or Agilent 1260 HPLC coupled to an Agilent 6530 Accurate-Mass Q-TOF LC/MS in positive or negative modes. Column: Phenomenex C18, $100 \AA, 100 \times 4.6 \mathrm{~mm}, 5 \mu \mathrm{m}$; Flow-rate: $0.2 \mathrm{~mL} / \mathrm{min}$; Solvent A: water $0.1 \%$ formic acid, Solvent B: acetonitrile $0.1 \%$ formic acid, loading at $10 \% \mathrm{~B}$, increasing the solvent gradient to $100 \% \mathrm{~B}$ in $20 \mathrm{~min}$, and then re-equilibrating the HPLC column over $7 \mathrm{~min}$ in $10 \% \mathrm{~B}$. The molecular weights of all the isolated compounds were obtained through LC/MS analysis.

\subsection{NMR Experiments}

NMR spectra including 1D (one dimension) and 2D (two dimensions) experiments were recorded in acetone- $d_{6}$ or $\mathrm{MeOH}-d_{4}$ or $\mathrm{CDCl}_{3}$ or DMSO- $d_{6}$ on a Bruker $400 \mathrm{MHz}$ NMR, which plays a major role in the structural determination of the isolated compounds.

\subsection{Analysis of Metals, Amino Acids, and Total Polysaccharides}

Iron (GB 5009, 90-2016), zinc (GB 5009, 14-2017), calcium (GB 5009, 92-2016), and amino acids (GB 5009, 124-2016) were analyzed according to the methods as described in the National Food Safety Standards, People's Republic of China. The concentration of total polysaccharides was measured according to the methods as published in the literatures [18]. 


\subsection{Cell Culture and Condition}

Nrf2 Antioxidant Pathway ARE Reporter-Hep G2 cell line was purchased from BPS Bioscience (San Diego, CA, USA). Cells were propagated at $37{ }^{\circ} \mathrm{C}$ in a humidified incubator with $5 \% \mathrm{CO}_{2}$, in Eagle's minimum essential medium (MEM, Corning, New York, NY, USA) with non-essential amino acids and supplemented with 10\% fetal bovine serum (FBS, Invitrogen, Waltham, MA, USA), penicillin and streptomycin (Thermo Fisher, Waltham, MA, USA). Cells were trypsinized and split every 6 to 7 days.

\subsection{Chemicals Exposure and Luciferase Assay to Measure the Nrf2 Activation}

Sterile DMSO (dimethyl sulfoxide) stock solutions of crude, HP20 fractions and DL-sulforaphane (Sigma \# S4441) were prepared in DMSO. The HepG2-Nrf2 stable cell line was seeded into 96-well plates at $4 \times 10^{4}$ per well in a final volume of $100 \mu \mathrm{L}$ MEM. $24 \mathrm{~h}$ after seeding, media was replaced with fresh MEM and the cells were treated with the crude extract or fractions or pure compounds. Plates were incubated for $24 \mathrm{~h}$, then $100 \mu \mathrm{L}$ ONE-Step Luciferase reagent (BPS Bioscience) was added to each well and the assay was performed according to manufacturer's instructions. Luminescence was detected using a luminometer (LUMIstar Galaxy BMG, Offenburg, Germany) and data are expressed as relative luminescence units (RLU) emitted from total assays. DL-sulforaphane was used as a positive control at a concentration of $5 \mu \mathrm{M}$. All experiments were performed in triplicate.

\subsection{Cell Viability Assay}

Cell viability was assessed by methylthiazoltetrazolium (MTT) assay (Sigma-Aldrich, St Louis, MO, USA) according to the manufacturer's instruction. Briefly, cells $\left(4 \times 10^{4}\right)$ were seeded into a 96-well plate in $100 \mu \mathrm{L}$ MEM and allowed to adhere overnight. Culture medium was replaced, and cells were treated with crude or fractionated samples (Fr. I-V) or pure compounds for $24 \mathrm{~h}$ treatments. The medium of each well was replaced by $200 \mu \mathrm{L}$ fresh medium plus $50 \mu \mathrm{L}$ of the MTT solution $\left(5 \mathrm{mg} / \mathrm{mL}\right.$ in PBS). The plates were incubated at $37^{\circ} \mathrm{C}$ for $4 \mathrm{~h}$. The absorbance being proportional to cell was subsequently measured at $570 \mathrm{~nm}$ in each well using a Bio-Rad 680 plate reader (Hercules, CA, USA). DL-sulforaphane was also used as a control at a concentration of $5 \mu \mathrm{M}$. All experiments were performed in triplicate.

\subsection{Statistical Analysis}

Values are expressed as the mean \pm standard error of the mean $p$ values $<0.05$ were considered statistically significant. All analyses were performed with the Student $t$-test using GraphPad Prism 5.1 (GraphPad, La Jolla, CA, USA).

\section{Results}

\subsection{Isolation and Structure Elucidation of Minor Compounds from Jing Liqueur}

In order to analyze the minor compounds in Jing liqueur, we concentrated $160 \mathrm{~L}$ of Jing liqueur Brand "Xiaoqu white liqueur," and separated the extract with HP20 (See Section 2.4 and Scheme 1) into five fractions (Fraction I: $100 \% \mathrm{H}_{2} \mathrm{O}$; Fraction II: $20 \% \mathrm{MeOH} / \mathrm{H}_{2} \mathrm{O}$; Fraction III: $50 \% \mathrm{MeOH} / \mathrm{H}_{2} \mathrm{O}$; Fraction IV: $80 \% \mathrm{MeOH} / \mathrm{H}_{2} \mathrm{O}$; Fraction V: $100 \% \mathrm{MeOH}$ ). Each of the fractions (II-V) was further separated with $\mathrm{C} 18$ preparative HPLC (Fraction II: 5-25\% $\mathrm{MeOH} / \mathrm{H}_{2} \mathrm{O}$ in $42 \mathrm{~min}$; Fraction III: 15-42\% $\mathrm{MeOH} / \mathrm{H}_{2} \mathrm{O}$ in 42 min; Fraction IV: $23-80 \% \mathrm{MeOH} / \mathrm{H}_{2} \mathrm{O}$ in 50 min; Fraction V: $50-10 \% \mathrm{MeOH} / \mathrm{H}_{2} \mathrm{O}$ in $56 \mathrm{~min}$ ), and subfractions (one subfraction per $\mathrm{min}$ ) were collected. Then the subfractions were purified with semi-preparative HPLC to get the pure compounds (See Tables 1-3 for retention times of the 189 pure compounds and semi-preparative HPLC conditions including columns, flow-rates, and solvent systems). In total, one hundred eighty nine (189) compounds, including flavonoids, terpenoids, alkaloids, coumarins, cinnamic acid or coumaric acid, and phenyl ethanol (or acetic acid) derivatives, 
benzoquinone, naphthoquinone, anthraquinones or phenanphrene derivatives, xanthones, chromone, and $\gamma$-pyrone derivatives, lignans, other aromatic compounds, and others were isolated and identified. The chemical structures were determined by using LC-MS and NMR as shown in Figures 1 and 2. The HPLC conditions, molecular formulas, sources of the corresponding plants, and references of the characterized compounds are summarized in Tables 1-3. All the MS, NMR spectra, and references for the 189 compounds isolated from Jing liqueur are listed in the Supplementary Material. These 189 compounds were simply categorized into nine classes.
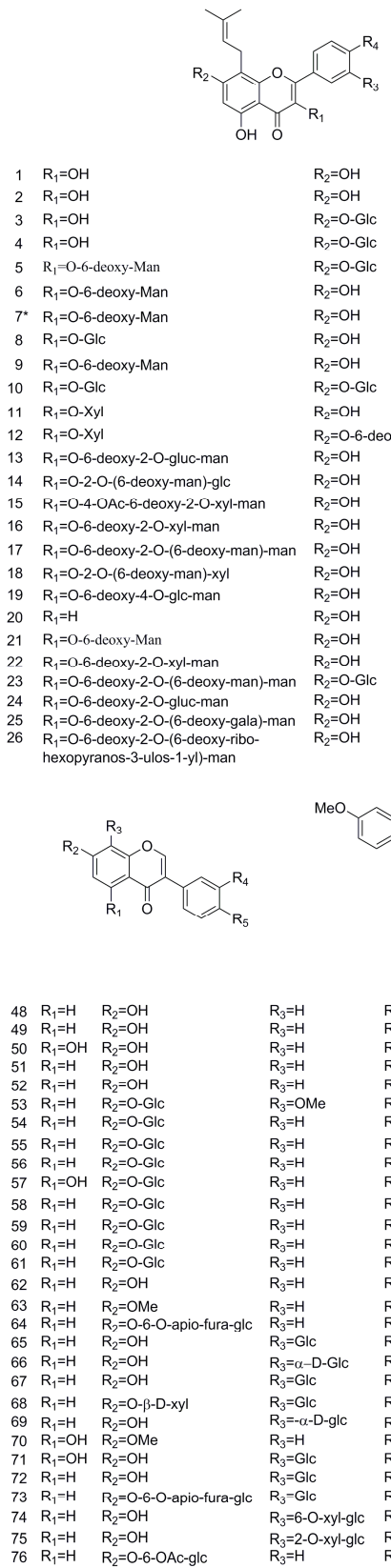

\begin{tabular}{|c|c|c|}
\hline $\mathrm{R}_{2}=\mathrm{OH}$ & $\mathrm{R}_{3}=\mathrm{H}$ & $\mathrm{R}_{4}=\mathrm{OH}$ \\
\hline $\mathrm{R}_{2}=\mathrm{OH}$ & $R_{3}=3-m e t h y|b u t-2-e n-1-y|$ & $\mathrm{R}_{4}=\mathrm{OH}$ \\
\hline$R_{2}=O-G l c$ & $\mathrm{R}_{3}=\mathrm{H}$ & $\mathrm{R}_{4}=\mathrm{OH}$ \\
\hline$R_{2}=O-G l c$ & $\mathrm{R}_{3}=\mathrm{H}$ & $\mathrm{R}_{4}=\mathrm{OMe}$ \\
\hline$R_{2}=0-G / c$ & $\mathrm{R}_{3}=\mathrm{H}$ & $\mathrm{R}_{4}=\mathrm{OMe}$ \\
\hline $\mathrm{R}_{2}=\mathrm{OH}$ & $\mathrm{R}_{3}=\mathrm{OH}$ & $\mathrm{R}_{4}=\mathrm{OMe}$ \\
\hline $\mathrm{R}_{2}=\mathrm{OH}$ & $\mathrm{R}_{3}=\mathrm{OH}$ & $\mathrm{R}_{4}=\mathrm{OH}$ \\
\hline $\mathrm{R}_{2}=\mathrm{OH}$ & $\mathrm{R}_{3}=\mathrm{H}$ & $\mathrm{R}_{4}=\mathrm{OH}$ \\
\hline $\mathrm{R}_{2}=\mathrm{OH}$ & $\mathrm{R}_{3}=\mathrm{H}$ & $\mathrm{R}_{4}=\mathrm{OH}$ \\
\hline$R_{2}=O-G l c$ & $\mathrm{R}_{3}=\mathrm{H}$ & $\mathrm{R}_{4}=\mathrm{OH}$ \\
\hline $\mathrm{R}_{2}=\mathrm{OH}$ & $\mathrm{R}_{3}=\mathrm{H}$ & $\mathrm{R}_{4}=\mathrm{OMe}$ \\
\hline$R_{2}=0-6$-deoxy-Man & $\mathrm{R}_{3}=\mathrm{H}$ & $\mathrm{R}_{4}=\mathrm{OMe}$ \\
\hline $\mathrm{R}_{2}=\mathrm{OH}$ & $\mathrm{R}_{3}=\mathrm{H}$ & $\mathrm{R}_{4}=\mathrm{OH}$ \\
\hline $\mathrm{R}_{2}=\mathrm{OH}$ & $\mathrm{R}_{3}=\mathrm{H}$ & $\mathrm{R}_{4}=\mathrm{OH}$ \\
\hline $\mathrm{R}_{2}=\mathrm{OH}$ & $\mathrm{R}_{3}=\mathrm{H}$ & $\mathrm{R}_{4}=\mathrm{OMe}$ \\
\hline $\mathrm{R}_{2}=\mathrm{OH}$ & $\mathrm{R}_{3}=\mathrm{H}$ & $\mathrm{R}_{4}=\mathrm{OH}$ \\
\hline $\mathrm{R}_{2}=\mathrm{OH}$ & $\mathrm{R}_{3}=\mathrm{H}$ & $\mathrm{R}_{4}=\mathrm{OMe}$ \\
\hline $\mathrm{R}_{2}=\mathrm{OH}$ & $\mathrm{R}_{3}=\mathrm{H}$ & $\mathrm{R}_{4}=\mathrm{OMe}$ \\
\hline $\mathrm{R}_{2}=\mathrm{OH}$ & $\mathrm{R}_{3}=\mathrm{H}$ & $\mathrm{R}_{4}=\mathrm{OMe}$ \\
\hline $\mathrm{R}_{2}=\mathrm{OH}$ & $R_{3}=3$-methylbut-2-en-1-yl & $\mathrm{R}_{4}=\mathrm{OH}$ \\
\hline $\mathrm{R}_{2}=\mathrm{OH}$ & $\mathrm{R}_{3}=\mathrm{H}$ & $\mathrm{R}_{4}=\mathrm{OMe}$ \\
\hline $\mathrm{R}_{2}=\mathrm{OH}$ & $\mathrm{R}_{3}=\mathrm{H}$ & $\mathrm{R}_{4}=\mathrm{OMe}$ \\
\hline$R_{2}=0-G l c$ & $\mathrm{R}_{3}=\mathrm{H}$ & $\mathrm{R}_{4}=\mathrm{OMe}$ \\
\hline $\mathrm{R}_{2}=\mathrm{OH}$ & $\mathrm{R}_{3}=\mathrm{H}$ & $\mathrm{R}_{4}=\mathrm{OMe}$ \\
\hline $\mathrm{R}_{2}=\mathrm{OH}$ & $\mathrm{R}_{3}=\mathrm{H}$ & $\mathrm{R}_{4}=\mathrm{OMe}$ \\
\hline $\mathrm{R}_{2}^{2}=\mathrm{OH}$ & $\mathrm{R}_{3}=\mathrm{H}$ & $\mathrm{R}_{4}=\mathrm{OMe}$ \\
\hline
\end{tabular}

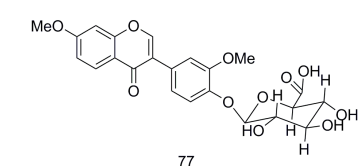

77

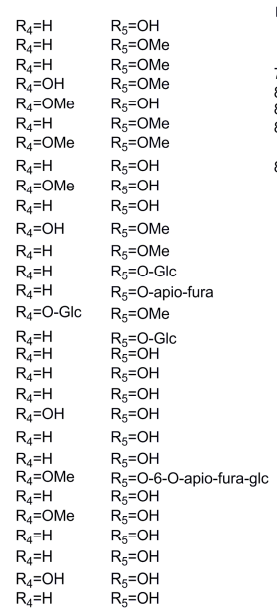

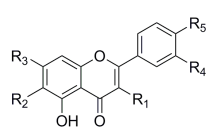

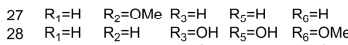

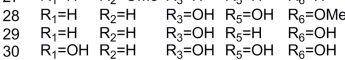
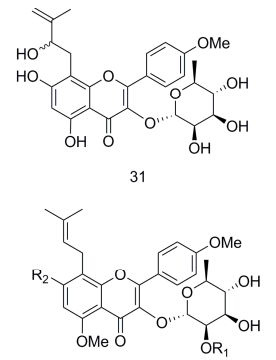

$32 \quad \mathrm{R}_{1}=\mathrm{H}$ $R_{2}=O-G l c$
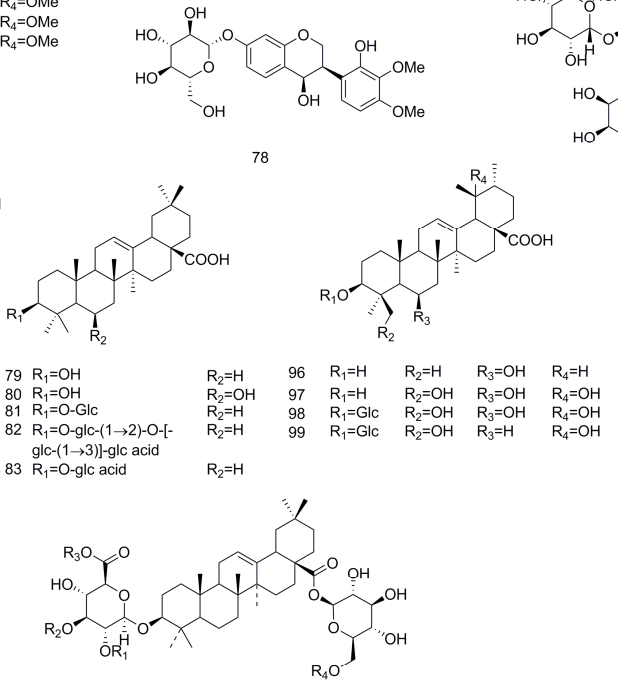

$\begin{array}{ll}85 & R_{1}=H \\ 86 & R_{1}=H \\ 87 & R_{1}=H\end{array}$

$\begin{array}{ll}88 & \mathrm{R}_{1}=\mathrm{Glc} \\ 89 & \mathrm{R}_{1}=\mathrm{Glc}\end{array}$

$\begin{array}{ll}89 & R_{1}=G l c \\ 90 & R_{1}=G l c \\ 91 & R_{1}=\wedge \text { Arab } \\ 92 & R_{1}=\text { Arab } \\ 93 & R_{1}=\text { Ara }\end{array}$

$\begin{array}{ll}92 & R_{1}=A r a b \\ 93 & R_{1}=H\end{array}$

$\begin{array}{ll}R_{1}=H \\ 94 & R_{1}=6-\text { deoxy-man } \\ 95 & R_{1}=H\end{array}$
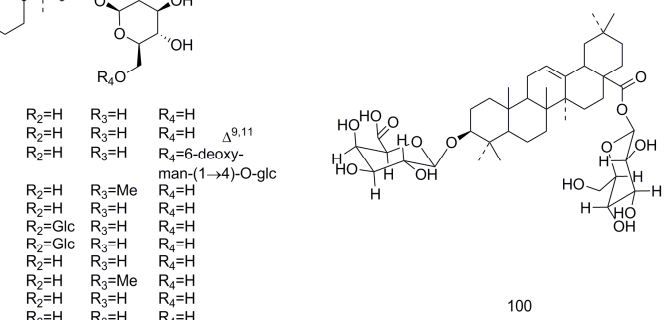

100

Figure 1. Structures of compounds 1-100 isolated from Jing liqueur. 

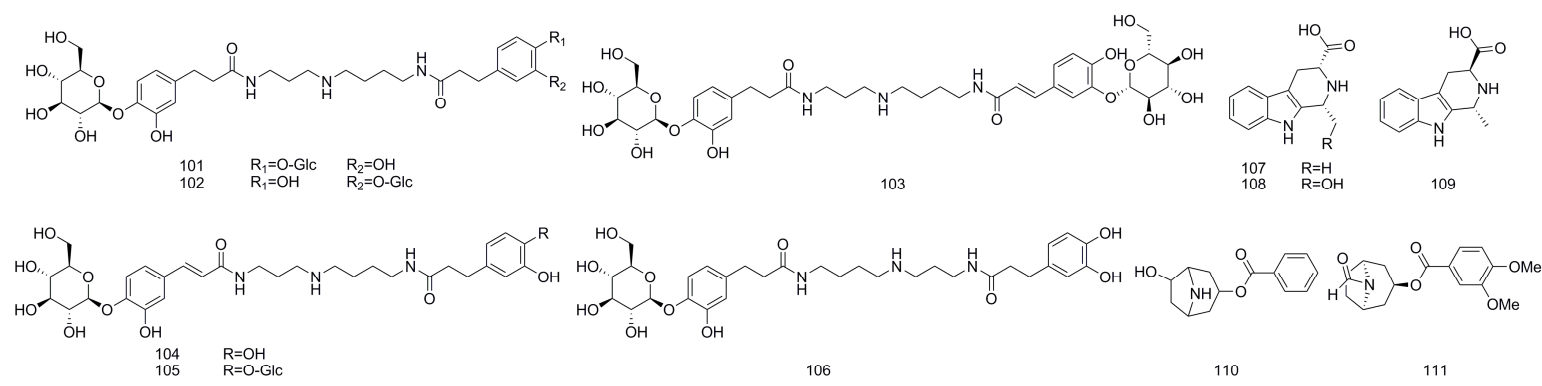

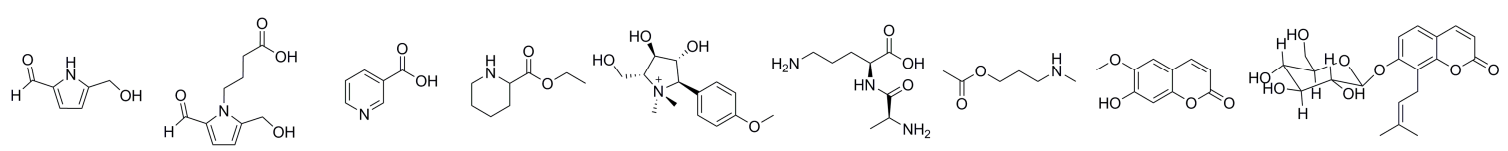

112

113

114

$\begin{array}{lll}116 & 117 & 118\end{array}$

120

(OHOH

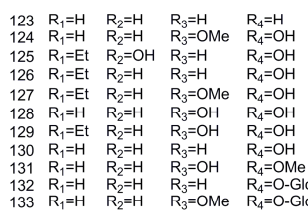

$\begin{array}{ll}140 & R=H \\ 141 & R=M e\end{array}$

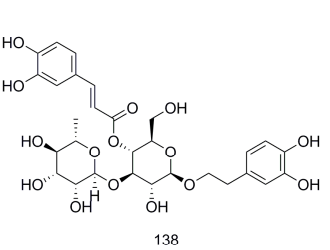

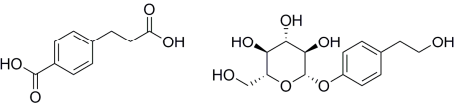

134
管

139

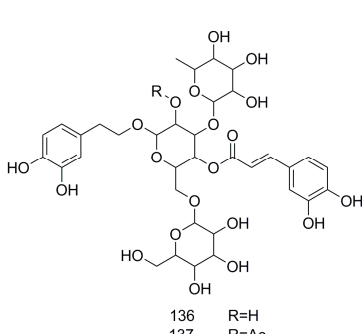

$\begin{array}{ll}136 & R=H \\ 137 & R=A C\end{array}$

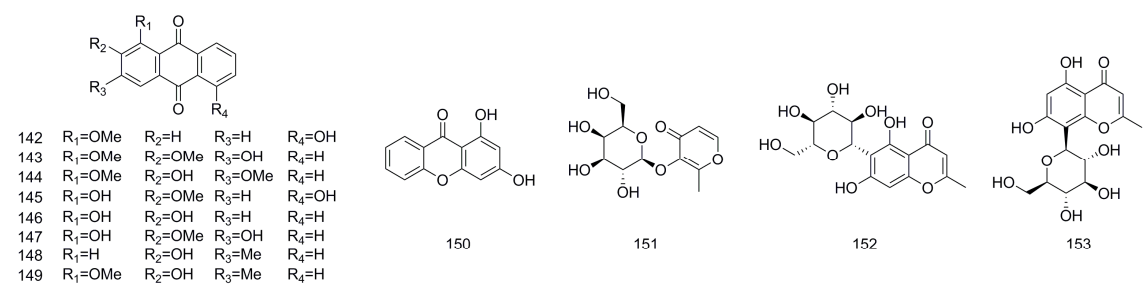<smiles>Cc1cc(=O)c2c(O)cc(OC3OC(CO)C(O)[C@H](O)C3O)cc2o1</smiles><smiles>COc1cc2occc(=O)c2cc1OC1OC(CO)C(O)C(O)C1O</smiles>
OेH

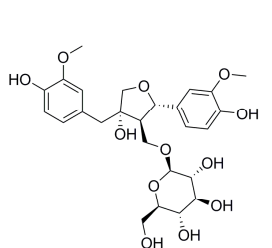

156

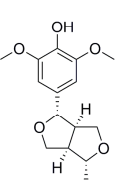<smiles>COc1cccc(OC)c1O</smiles>

157
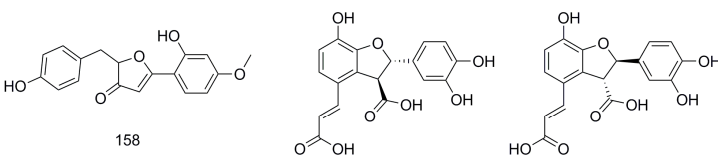

159

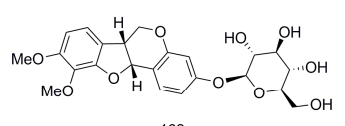

162

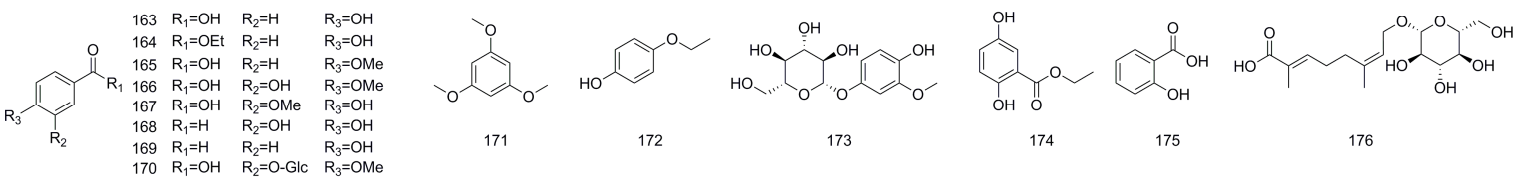

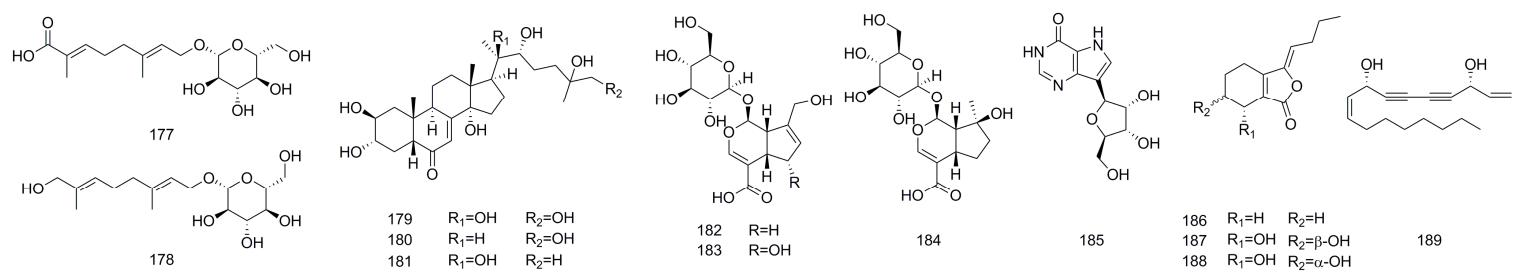

Figure 2. Structures of compounds 101-189 isolated from Jing liqueur.

(1). Flavonoids: Seventy-eight flavonoids (\#1-78, Table 1, Figure 1) have been isolated. They are either aglycones or glycosides of flavone, flavonol, flavanone, isoflavone, and isoflavanone derivatives. The new compound (7) was obtained as a light yellowish powder. Its molecular formula was determined as $\mathrm{C}_{26} \mathrm{H}_{28} \mathrm{O}_{11}$ based on NMR and high resolution ESI mass spectrometry (HRESIMS) data $(\mathrm{m} / \mathrm{z} 517.1710$ 
$[\mathrm{M}+\mathrm{H}]^{+}$, calcd for $\left.\mathrm{C}_{26} \mathrm{H}_{29} \mathrm{O}_{11}, 517.1710\right)$. Its ${ }^{1} \mathrm{H}$ NMR spectrum in $\mathrm{CD}_{3} \mathrm{OD}$ exhibited signals similar to those of caohuoside $C$ (6) [19]. The only difference between 6 and 7 was the presence of the ${ }^{1} \mathrm{H}$ NMR signal for a methoxy group at $4^{\prime}$-position in 6 , but absence in 7 . Hence, the new compound (7) was determined as 4'-O-demethyl caohuoside C (Table 1 and Table S1, Figure 1 and Figure S1). (2). Terpenoids: Twenty-two triterpenoids (\#79-100) have been isolated from Jing liqueur (Table 2, Figure 1). These compounds are either simple oleanane or ursane derivatives, or glycosides of oleanolic acid. The sugars were connected to either 3-position, or 28-position, or both of the aglycones. Eighteen of these twenty-two triterpenoids are saponins, among which seventeen are oleanane glycosides and one is ursane glycoside [20]. (3). Alkaloids: Eighteen alkaloids including derivatives of spermidine, indole, tropane, pyrrole, piperidine, and alanyllysine (\#101-118) have been isolated from Jing liqueur (Table 2, Figure 2). (4). Coumarins, cinnamic acid or coumaric acid, and phenyl ethanol (or acetic acid) derivatives: Twenty-one coumarins, cinnamic acid or coumaric acid, and phenyl ethanol (or acetic acid) derivatives (\#119-139) were isolated from Jing liqueur (Table 2, Figure 2), including ten cinnamic acid analogs. (5). Benzoquinone, naphthoquinone, anthraquinones, or phenanphrene derivatives: Ten benzoquinone, naphthoquinone, anthraquinones, or phenanphrene derivatives (\#140-149) were isolated from Jing liqueur (Table 3, Figure 2), eight of which were anthraquinones. (6). Xanthones, chromone, and $\gamma$-pyrone derivatives: Six xanthones, chromone, and $\gamma$-pyrone derivatives (\#150-155) were isolated (Table 3 , Figure 2). (7). Lignans: Seven lignans (\#156-162) were isolated (Table 3, Figure 2). (8). Other aromatic compounds: Thirteen small aromatic compounds (\#163-175) were isolated (Table 3, Figure 2). They were either benzoic acid or phenolic derivatives. (9). Other compounds: Fourteen other compounds (\#176-189) were isolated, including three monoterpenoid glycosides, three cholesterol analogs, three iridoid glycosides, one nucleoside analog, three $\gamma$-lactone, and one alkyne derivatives (Table 3, Figure 2). In summary, one hundred eighty nine (189) compounds have been isolated, including ninety two (92) from fraction V, fifty seven (57) from fraction IV, thirty four (34) from fraction III, and six (6) from fraction II.

Table 1. Flavanoids (\#1-78) isolated from Jing liqueur.

\begin{tabular}{|c|c|c|c|c|c|}
\hline NO & Fr. & $t_{\mathrm{R}}$, HPLC Condition & MF & Compound Name & Reference (See SM) \\
\hline 1 & $\mathrm{~V}$ & C.1, ACN, 33\% & $\mathrm{C}_{20} \mathrm{H}_{18} \mathrm{O}_{6}$ & Noranhydroicaritin & Komatsu et al. 1970 \\
\hline 2 & $\mathrm{~V}$ & Sepherdex LH20 & $\mathrm{C}_{25} \mathrm{H}_{26} \mathrm{O}_{6}$ & Broussoflavonol F & Fang et al. 1995 \\
\hline 3 & IV & $\begin{array}{c}71 \mathrm{~min}, \mathrm{C} .1, \mathrm{ACN}, 18 \% \\
110 \mathrm{~min}\end{array}$ & $\mathrm{C}_{26} \mathrm{H}_{28} \mathrm{O}_{11}$ & Epimedoside C & Li et al. 1990 \\
\hline 4 & IV & $\begin{array}{c}68 \mathrm{~min}, \mathrm{C} .2, \mathrm{ACN}, 30-35 \%, \\
80 \mathrm{~min}\end{array}$ & $\mathrm{C}_{27} \mathrm{H}_{30} \mathrm{O}_{11}$ & Icariside I & Mizuno et al. 1987 \\
\hline 5 & $\mathrm{~V}$ & $56 \mathrm{~min}, \mathrm{C} .1, \mathrm{ACN}, 24 \%$ & $\mathrm{C}_{33} \mathrm{H}_{40} \mathrm{O}_{15}$ & Ieariline & Liang et al. 1988 \\
\hline 8 & $\mathrm{~V}$ & C. $1, \mathrm{ACN}, 35 \%$ & $\mathrm{C}_{26} \mathrm{H}_{28} \mathrm{O}_{11}$ & Phelodendrozide & Wang et al. 2010 \\
\hline 9 & $\mathrm{~V}$ & C. $1, \mathrm{ACN}, 33 \%$ & $\mathrm{C}_{26} \mathrm{H}_{28} \mathrm{O}_{10}$ & baohuoside II & Dong et al. 1994 \\
\hline 10 & IV & $\begin{array}{c}53.5 \mathrm{~min}, \mathrm{C} .2, \mathrm{ACN}, 17 \% \\
100 \mathrm{~min}\end{array}$ & $\mathrm{C}_{32} \mathrm{H}_{38} \mathrm{O}_{16}$ & Hexandraside E & Leu et al. 2006 \\
\hline 11 & $\mathrm{~V}$ & C. $2, \mathrm{ACN}, 40-50 \%, 48 \mathrm{~min}$ & $\mathrm{C}_{26} \mathrm{H}_{28} \mathrm{O}_{10}$ & & \\
\hline 12 & $\mathrm{~V}$ & $49 \min$, C. 2, ACN, $34 \%$ & $\mathrm{C}_{32} \mathrm{H}_{38} \mathrm{O}_{14}$ & Baohuoside IV & Li and Liu 1990 \\
\hline 17 & IV & $\begin{array}{c}75 \mathrm{~min}, \mathrm{C} .2, \mathrm{ACN}, 30-35 \% \\
80 \mathrm{~min}\end{array}$ & $\mathrm{C}_{33} \mathrm{H}_{40} \mathrm{O}_{14}$ & & Ueda et al. 1992 \\
\hline 18 & $\mathrm{~V}$ & $35.5 \mathrm{~min}, \mathrm{C} .2 \mathrm{ACN}, 36 \%$ & $\mathrm{C}_{32} \mathrm{H}_{38} \mathrm{O}_{14}$ & & Zhao et al. 2010 \\
\hline 19 & $\mathrm{~V}$ & $39 \mathrm{~min}, \mathrm{C} .2, \mathrm{ACN}, 34 \%$ & $\mathrm{C}_{33} \mathrm{H}_{40} \mathrm{O}_{15}$ & Baohuoside VII & Li et al. 1988 \\
\hline 20 & $\mathrm{~V}$ & Sepherdex LH20 & $\mathrm{C}_{25} \mathrm{H}_{26} \mathrm{O}_{5}$ & $5,7,4^{\prime}$-trihydroxy-8, 3'-diprenylflavone & Guo et al. 2006 \\
\hline 21 & $\mathrm{~V}$ & C. $1, \mathrm{MeOH}, 65 \%$ & $\mathrm{C}_{27} \mathrm{H}_{30} \mathrm{O}_{10}$ & Icariside II & Zhang et al. 2006 \\
\hline 22 & $\mathrm{~V}$ & C.1, $\mathrm{MeOH}, 65 \%$ & $\mathrm{C}_{32} \mathrm{H}_{38} \mathrm{O}_{14}$ & Sagittatoside B & Mizuno et al. 1988 \\
\hline 23 & $\mathrm{~V}$ & C. $1, \mathrm{ACN}, 24 \%$ & $\mathrm{C}_{39} \mathrm{H}_{50} \mathrm{O}_{19}$ & Epimedin C & Mizuno et al. 1988 \\
\hline 24 & $\mathrm{~V}$ & C. $1, \mathrm{ACN}, 24 \%$ & $\mathrm{C}_{33} \mathrm{H}_{40} \mathrm{O}_{15}$ & Sagittatoside A & Mizuno et al. 1988 \\
\hline 25 & $\mathrm{~V}$ & C. $1, \mathrm{MeOH}, 65 \%$ & $\mathrm{C}_{33} \mathrm{H}_{40} \mathrm{O}_{14}$ & 2"-O-rhamnosyl icariside II & Zhao et al. 2016 \\
\hline 26 & $\mathrm{~V}$ & C. $1, \mathrm{MeOH}, 64 \%$ & $\mathrm{C}_{33} \mathrm{H}_{38} \mathrm{O}_{14}$ & $3^{\prime \prime \prime}$-carbonyl-2"- $\beta$-L-quinovosyl icariside II & Zhang et al. 2006 \\
\hline 27 & $\mathrm{~V}$ & C.1, $\mathrm{ACN}, 33 \%$ & $\mathrm{C}_{16} \mathrm{H}_{12} \mathrm{O}_{4}$ & & Asahina et al. 1935 \\
\hline
\end{tabular}


Table 1. Cont.

\begin{tabular}{|c|c|c|c|c|c|}
\hline NO & Fr. & $t_{\mathrm{R}}$, HPLC Condition & MF & Compound Name & Reference (See SM) \\
\hline 28 & IV & $\begin{array}{c}85.5 \mathrm{~min}, \mathrm{C} .1, \mathrm{ACN}, 18 \% \\
110 \mathrm{~min}\end{array}$ & $\mathrm{C}_{39} \mathrm{H}_{50} \mathrm{O}_{20}$ & & Das and Tripathi 2002 \\
\hline 29 & $\mathrm{~V}$ & Sepherdex LH20 & $\mathrm{C}_{15} \mathrm{H}_{10} \mathrm{O}_{5}$ & Versulin & Geissman et al. 1946 \\
\hline 30 & $\mathrm{~V}$ & Sepherdex LH20 & $\mathrm{C}_{15} \mathrm{H}_{10} \mathrm{O}_{7}$ & Xanthaurine & Bao et al. 2004 \\
\hline 31 & $\mathrm{~V}$ & C. $1, \mathrm{ACN}, 35 \%$ & $\mathrm{C}_{27} \mathrm{H}_{30} \mathrm{O}_{11}$ & Koreanoside E & Li et al. 2015 \\
\hline 32 & $\mathrm{~V}$ & C. $1, \mathrm{MeOH}, 64 \%$ & $\mathrm{C}_{34} \mathrm{H}_{42} \mathrm{O}_{15}$ & & Hu et al. 2010 \\
\hline 33 & $\mathrm{~V}$ & C. $1, \mathrm{MeOH}, 64 \%$ & $\mathrm{C}_{34} \mathrm{H}_{42} \mathrm{O}_{14}$ & & \\
\hline 34 & $\mathrm{~V}$ & C. $1, \mathrm{ACN}, 24 \%$ & $\mathrm{C}_{39} \mathrm{H}_{50} \mathrm{O}_{20}$ & Epimedin A & Han, Lee 2017 \\
\hline 35 & $\mathrm{~V}$ & C.1, ACN, $24 \%$ & $\mathrm{C}_{39} \mathrm{H}_{50} \mathrm{O}_{20}$ & Maohuoside B & Li et al. 2006 \\
\hline 36 & $\mathrm{~V}$ & C. $1, \mathrm{ACN}, 24 \%$ & $\mathrm{C}_{38} \mathrm{H}_{48} \mathrm{O}_{19}$ & Epimedin B & Guo and Xiao 2003 \\
\hline 37 & $\mathrm{~V}$ & C. $1, \mathrm{ACN}, 24 \%$ & $\mathrm{C}_{39} \mathrm{H}_{50} \mathrm{O}_{19}$ & Hexandraside D & Mizuno et al. 1991 \\
\hline 38 & $\mathrm{~V}$ & C. $1, \mathrm{ACN}, 28 \%$ & $\mathrm{C}_{39} \mathrm{H}_{48} \mathrm{O}_{19}$ & & Zhao et al. 2008 \\
\hline 39 & $\mathrm{~V}$ & C. $1, \mathrm{ACN}, 28 \%$ & $\mathrm{C}_{39} \mathrm{H}_{50} \mathrm{O}_{19}$ & & Ueda et al. 1992 \\
\hline 40 & $\mathrm{~V}$ & $\begin{array}{c}29 \mathrm{~min}, \mathrm{C} .1, \mathrm{ACN}, 22-33 \% \\
60 \mathrm{~min}\end{array}$ & $\mathrm{C}_{39} \mathrm{H}_{50} \mathrm{O}_{20}$ & Hexandraside F & Wang et al. 2007 \\
\hline 41 & $\mathrm{~V}$ & $\begin{array}{c}33 \mathrm{~min}, \mathrm{C} .1, \mathrm{ACN}, 22-33 \% \\
60 \mathrm{~min}\end{array}$ & $\mathrm{C}_{38} \mathrm{H}_{48} \mathrm{O}_{19}$ & & Zhao et al. 2010 \\
\hline 42 & IV & $\begin{array}{c}85.5 \mathrm{~min}, \mathrm{C} .2, \mathrm{ACN}, 18 \% \\
110 \mathrm{~min}\end{array}$ & $\mathrm{C}_{16} \mathrm{H}_{12} \mathrm{O}_{6}$ & Diosmetin & Takeda et al. 2007 \\
\hline 3 & $\mathrm{~V}$ & $\begin{array}{c}77.5 \mathrm{~min}, \mathrm{C} .2, \mathrm{ACN} \\
16-20 \%, 100 \mathrm{~min}\end{array}$ & $\mathrm{C}_{38} \mathrm{H}_{48} \mathrm{O}_{20}$ & Rouhuoside & Li et al. 1990 \\
\hline 44 & $\mathrm{~V}$ & $\begin{array}{c}47 \mathrm{~min}, \mathrm{C} .2, \mathrm{ACN}, 22-33 \% \\
60 \mathrm{~min}\end{array}$ & $\mathrm{C}_{38} \mathrm{H}_{48} \mathrm{O}_{20}$ & Diphylloside A/Ikarisoside C & Jia et al. 1998 \\
\hline 45 & IV & $\begin{array}{c}39 \mathrm{~min}, \mathrm{C} .2, \mathrm{MeOH} \\
50-60 \% 80 \mathrm{~min}\end{array}$ & $\mathrm{C}_{39} \mathrm{H}_{48} \mathrm{O}_{21}$ & & Jin et al. 2013 \\
\hline 46 & $\mathrm{~V}$ & C. $1, \mathrm{ACN}, 28 \%$ & $\mathrm{C}_{39} \mathrm{H}_{48} \mathrm{O}_{20}$ & & Jin et al. 2013 \\
\hline 47 & III & $\begin{array}{c}41.5 \mathrm{~min}, \mathrm{C} .1, \mathrm{MeOH}, \\
6-8.5 \%, 65 \mathrm{~min}\end{array}$ & $\mathrm{C}_{34} \mathrm{H}_{44} \mathrm{O}_{20}$ & & Li et al. 2012 \\
\hline 48 & $\mathrm{~V}$ & $\begin{array}{l}38.5 \mathrm{~min}, \mathrm{C} .2, \mathrm{ACN} \\
22-24 \%, 75 \mathrm{~min}\end{array}$ & $\mathrm{C}_{15} \mathrm{H}_{10} \mathrm{O}_{4}$ & Isoaurostatin/4',7-Dihydroxyisoflavone & Xu et al. 1979 \\
\hline 49 & $\mathrm{~V}$ & Sepherdex LH20 & $\mathrm{C}_{16} \mathrm{H}_{12} \mathrm{O}_{4}$ & Formononetin & Reiners 1966 \\
\hline 50 & $\mathrm{~V}$ & $\begin{array}{c}47 \mathrm{~min}, \mathrm{C} .2, \mathrm{ACN}, 22-24 \% \\
75 \mathrm{~min}\end{array}$ & $\mathrm{C}_{16} \mathrm{H}_{12} \mathrm{O}_{5}$ & Biochanin A, Olmelin & Nilsson 1961 \\
\hline 51 & $\mathrm{~V}$ & $\begin{array}{c}47 \mathrm{~min}, \mathrm{C} .2, \mathrm{ACN}, 22-24 \% \\
75 \mathrm{~min}\end{array}$ & $\mathrm{C}_{16} \mathrm{H}_{12} \mathrm{O}_{5}$ & Calycosin, Cyclosin & Markham et al. 1968 \\
\hline 52 & $\mathrm{~V}$ & $\begin{array}{c}\text { C. } 2, \mathrm{ACN}, 20-22 \%, 75 \mathrm{~min} \\
55.5 \mathrm{~min}\end{array}$ & $\mathrm{C}_{16} \mathrm{H}_{12} \mathrm{O}_{5}$ & 7,4'-Dihydroxy-3'-methoxyisoflavone & Hirakura et al. 1997 \\
\hline 53 & $\mathrm{~V}$ & $\begin{array}{c}\text { C. } 2, \mathrm{ACN}, 20-22 \%, 75 \mathrm{~min} \\
55.5 \mathrm{~min}\end{array}$ & $\mathrm{C}_{23} \mathrm{H}_{24} \mathrm{O}_{10}$ & 8-O-Methylretusin-7-O- $\beta$-D-glucopyranoside & Rukachaisirikul 2002 \\
\hline 54 & $\mathrm{~V}$ & $\begin{array}{c}69 \mathrm{~min}, \mathrm{C} .1, \mathrm{MeOH}, 35 \% \\
80 \mathrm{~min}\end{array}$ & $\mathrm{C}_{23} \mathrm{H}_{24} \mathrm{O}_{10}$ & & Clarke et al. 2004 \\
\hline 55 & IV & $\begin{array}{c}17 \mathrm{~min}, \mathrm{C} .2, \mathrm{ACN}, 15-18 \% \\
80 \mathrm{~min}\end{array}$ & $\mathrm{C}_{21} \mathrm{H}_{20} \mathrm{O}_{9}$ & Daidzoside & Xiao et al. 2016 \\
\hline 56 & IV & $\begin{array}{c}17 \mathrm{~min}, \mathrm{C} .2, \mathrm{ACN}, 15-18 \% \\
80 \mathrm{~min}\end{array}$ & $\mathrm{C}_{22} \mathrm{H}_{22} \mathrm{O}_{10}$ & 3'-Methoxydaidzin & Hirakura et al. 1997 \\
\hline 57 & IV & $\begin{array}{c}38 \mathrm{~min}, \mathrm{C} .2, \mathrm{ACN}, 20 \% \\
90 \mathrm{~min}\end{array}$ & $\mathrm{C}_{21} \mathrm{H}_{20} \mathrm{O}_{10}$ & Genistoside & Yuan et al. 2008 \\
\hline 58 & $\mathrm{~V}$ & $\begin{array}{c}66.5 \mathrm{~min}, \mathrm{C} .2, \mathrm{ACN} \\
22-24 \%, 75 \mathrm{~min}\end{array}$ & $\mathrm{C}_{22} \mathrm{H}_{22} \mathrm{O}_{10}$ & Calycosin 7-glucoside & Markham et al. 1968 \\
\hline 59 & IV & $\begin{array}{c}59 \mathrm{~min}, \mathrm{C} .1, \mathrm{ACN}, 18 \% \\
110 \mathrm{~min}\end{array}$ & $\mathrm{C}_{22} \mathrm{H}_{22} \mathrm{O}_{9}$ & Ononoside & Lebreton et al. 1967 \\
\hline 60 & IV & $14.5 \mathrm{~min}, \mathrm{C} .2, \mathrm{MeOH}, 20 \%$ & $\mathrm{C}_{27} \mathrm{H}_{30} \mathrm{O}_{14}$ & Daidzein $7,4^{\prime}$-diglucoside & Li et al. 2014 \\
\hline 61 & IV & $\begin{array}{c}33.5 \mathrm{~min}, \mathrm{C} .2, \mathrm{MeOH}, 28 \% \\
100 \mathrm{~min}\end{array}$ & $\mathrm{C}_{26} \mathrm{H}_{28} \mathrm{O}_{14}$ & Neobacin & Breytenbach 1986 \\
\hline 62 & IV & $\begin{array}{c}19 \mathrm{~min}, \mathrm{C} .2, \mathrm{ACN}, 15-18 \%, \\
80 \mathrm{~min}\end{array}$ & $\mathrm{C}_{22} \mathrm{H}_{22} \mathrm{O}_{10}$ & Caragiside B & Nisar et al. 2011 \\
\hline 63 & $\mathrm{~V}$ & C. $1, \mathrm{MeOH}, 35 \%, 75 \mathrm{~min}$ & $\mathrm{C}_{22} \mathrm{H}_{22} \mathrm{O}_{9}$ & Isoononin & Liu et al. 2005 \\
\hline 64 & IV & $24 \mathrm{~min}, \mathrm{C} .2, \mathrm{MeOH}, 20 \%$ & $\mathrm{C}_{26} \mathrm{H}_{28} \mathrm{O}_{14}$ & Ambocin & Breytenbach 1986 \\
\hline 65 & IV & C. $2, \mathrm{ACN}, 15-18 \%, 80 \mathrm{~min}$ & $\mathrm{C}_{21} \mathrm{H}_{20} \mathrm{O}_{9}$ & Neopuerarin & Zhang et al. 2009 \\
\hline 66 & IV & $\begin{array}{c}42 \mathrm{~min}, \mathrm{C} .2, \mathrm{ACN}, 20 \% \\
90 \mathrm{~min}\end{array}$ & $\mathrm{C}_{21} \mathrm{H}_{20} \mathrm{O}_{9}$ & & Ma et al. 2017 \\
\hline 67 & IV & $18 \mathrm{~min}, \mathrm{C} .2, \mathrm{MeOH}, 20 \%$ & $\mathrm{C}_{21} \mathrm{H}_{20} \mathrm{O}_{10}$ & 8-C-Glucosyl-7, $3^{\prime}, 4^{\prime}$-trihydroxy isoflavone & Wong et al. 2017 \\
\hline 68 & IV & $\begin{array}{l}63 \mathrm{~min}, \mathrm{C} .2, \mathrm{MeOH}, 20 \% \\
56 \mathrm{~min}, 20-30 \% 40 \mathrm{~min}\end{array}$ & $\mathrm{C}_{26} \mathrm{H}_{28} \mathrm{O}_{13}$ & & Chen et al. 2009 \\
\hline 69 & IV & C. $2, \mathrm{ACN}, 15-18 \%, 80 \mathrm{~min}$ & $\mathrm{C}_{21} \mathrm{H}_{20} \mathrm{O}_{9}$ & Neopuerarin A & Zhang et al. 2009 \\
\hline 70 & III & $\begin{array}{c}39 \mathrm{~min}, \mathrm{C} .2, \mathrm{MeOH}, \\
6-8.5 \%, 65 \mathrm{~min}\end{array}$ & $\mathrm{C}_{28} \mathrm{H}_{32} \mathrm{O}_{15}$ & & Wang et al. 2006 \\
\hline 71 & III & $\begin{array}{l}26 \mathrm{~min}, \mathrm{C} .2, \mathrm{MeOH}, 28 \%, \\
50 \mathrm{~min}\end{array}$ & $\mathrm{C}_{21} \mathrm{H}_{20} \mathrm{O}_{10}$ & & Pistelli et al. 1998 \\
\hline 72 & IV & $\begin{array}{l}54 \mathrm{~min}, \mathrm{C} .2, \mathrm{ACN}, 20 \% \\
90 \mathrm{~min}\end{array}$ & $\mathrm{C}_{22} \mathrm{H}_{22} \mathrm{O}_{10}$ & & Ohshima et al. 1988 \\
\hline 73 & IV & $\begin{array}{l}58.5 \mathrm{~min}, \mathrm{C} .2, \mathrm{ACN}, 20 \%, \\
90 \mathrm{~min}\end{array}$ & $\mathrm{C}_{26} \mathrm{H}_{28} \mathrm{O}_{13}$ & Puerarin apioside & Ingham et al. 1986 \\
\hline
\end{tabular}


Table 1. Cont.

\begin{tabular}{|c|c|c|c|c|c|}
\hline NO & Fr. & $t_{\mathrm{R}}$, HPLC Condition & MF & Compound Name & Reference (See SM) \\
\hline 74 & IV & $\begin{array}{c}67.5 \mathrm{~min}, \mathrm{C} .2, \mathrm{ACN}, 20 \%, \\
90 \mathrm{~min}\end{array}$ & $\mathrm{C}_{26} \mathrm{H}_{28} \mathrm{O}_{13}$ & & Kinjo et al. 1987 \\
\hline 75 & III & $\begin{array}{c}45 \mathrm{~min}, \mathrm{C} .2, \mathrm{MeOH} \\
6-8.5 \%, 65 \mathrm{~min}\end{array}$ & $\mathrm{C}_{26} \mathrm{H}_{28} \mathrm{O}_{14}$ & & Peng et al. 2011 \\
\hline 76 & IV & $\begin{array}{c}73 \mathrm{~min}, \mathrm{C} .2, \mathrm{MeOH}, 28 \% \\
100 \mathrm{~min}\end{array}$ & $\mathrm{C}_{23} \mathrm{H}_{22} \mathrm{O}_{10}$ & Acetyldaidzin & Ohta et al. 1979 \\
\hline 77 & IV & $\begin{array}{c}24 \mathrm{~min}, \mathrm{C} .2, \mathrm{ACN}, 15-18 \% \text {, } \\
80 \mathrm{~min}\end{array}$ & $\mathrm{C}_{23} \mathrm{H}_{22} \mathrm{O}_{11}$ & & Zhou et al. 2013 \\
\hline 78 & IV & $\begin{array}{c}74 \mathrm{~min}, \mathrm{C} .1, \mathrm{ACN}, 18 \% 110 \\
\min \end{array}$ & $\mathrm{C}_{23} \mathrm{H}_{28} \mathrm{O}_{11}$ & Astraganoside & Liu et al. 2007 \\
\hline
\end{tabular}

C.1: C18, $5 \mu \mathrm{m}, 250 \times 10 \mathrm{~mm}$, flow ( $3 \mathrm{~mL} / \mathrm{min})$; C.2: C8, $5 \mu, 250 \times 10 \mathrm{~mm}$, flow (3 mL/min), see Section 2.4; * new compound, MS and NMR data (Table S1 and Figures S2-S7).

Table 2. Terpenoids (\#79-100), alkaloids (\#101-118), coumarins, cinnamic acid or coumaric acid, and phenyl ethanol (or acetic acid) derivatives (\#119-139) isolated from Jing liqueur.

\begin{tabular}{|c|c|c|c|c|c|}
\hline NO & Fr. & $t_{\mathrm{R}}$, HPLC Condition & MF & Compound & Reference (See SM) \\
\hline 79 & $\mathrm{~V}$ & $\begin{array}{c}58 \mathrm{~min}, \mathrm{C} .2, \mathrm{ACN}, 57 \%, 30 \\
\min , 57-62 \% 20 \mathrm{~min} \\
62-70 \% 29 \mathrm{~min}\end{array}$ & $\mathrm{C}_{30} \mathrm{H}_{48} \mathrm{O}_{3}$ & Oleanolic acid & Tan et al. 2002 \\
\hline 80 & $\mathrm{~V}$ & $\begin{array}{c}26 \mathrm{~min}, \mathrm{C} .2, \mathrm{ACN}, 57 \%, 30 \\
\mathrm{~min}, 57-62 \% 20 \mathrm{~min} \\
62-70 \% 29 \mathrm{~min}\end{array}$ & $\mathrm{C}_{30} \mathrm{H}_{48} \mathrm{O}_{4}$ & Sumaresinolic acid & Chan et al. 1992 \\
\hline 81 & $\mathrm{~V}$ & C. $1, \mathrm{MeOH}, 72 \%$ & $\mathrm{C}_{36} \mathrm{H}_{58} \mathrm{O}_{8}$ & 3-O- $\beta$-Glc-oleanolic acid & Dubois et al. 1990 \\
\hline 82 & IV & $\begin{array}{l}34 \mathrm{~min}, \mathrm{C} .2, \mathrm{MeOH} \\
\quad 60-70 \%, 60 \mathrm{~min}\end{array}$ & $\mathrm{C}_{48} \mathrm{H}_{76} \mathrm{O}_{19}$ & Calendulaglycoside B & $\begin{array}{l}\text { Vidal-Ollivier et al. } \\
\qquad 1989\end{array}$ \\
\hline 83 & $\mathrm{~V}$ & C. $1, \mathrm{MeOH}, 72 \%$ & $\mathrm{C}_{36} \mathrm{H}_{56} \mathrm{O}_{9}$ & Calenduloside E & Zhang et al. 2013 \\
\hline 84 & $\mathrm{~V}$ & $\begin{array}{c}\text { C.2, ACN, } 30-35 \%, 60 \mathrm{~min}, \\
35-40 \% 20 \mathrm{~min}\end{array}$ & $\mathrm{C}_{48} \mathrm{H}_{74} \mathrm{O}_{18}$ & Papyrioside LG & \\
\hline 85 & $\mathrm{~V}$ & $\begin{array}{c}27 \mathrm{~min}, \mathrm{C} .1, \mathrm{ACN}, 35-42 \% \text {, } \\
80 \mathrm{~min}, 42-100 \% 5 \mathrm{~min}\end{array}$ & $\mathrm{C}_{42} \mathrm{H}_{66} \mathrm{O}_{14}$ & Chikusetsusaponin Iva & Yang et al. 1995 \\
\hline 86 & $\mathrm{~V}$ & $\begin{array}{l}\text { C.2, ACN, } 30-35 \%, 60 \mathrm{~min}, \\
35-40 \% 20 \mathrm{~min}\end{array}$ & $\mathrm{C}_{42} \mathrm{H}_{64} \mathrm{O}_{14}$ & & Kuroda et al. 2006 \\
\hline 87 & IV & $\begin{array}{l}36.5 \mathrm{~min}, \mathrm{C} .2, \mathrm{MeOH} \\
65-68 \%, 40 \mathrm{~min}\end{array}$ & $\mathrm{C}_{54} \mathrm{H}_{86} \mathrm{O}_{23}$ & Scheffleraside II & $\begin{array}{l}\text { Mshvildadze et al. } \\
\quad 2001\end{array}$ \\
\hline 88 & $\mathrm{~V}$ & $\begin{array}{l}\text { C. } 2, \text { ACN, } 30-35 \%, 60 \mathrm{~min}, \\
35-40 \% 20 \mathrm{~min}\end{array}$ & $\mathrm{C}_{49} \mathrm{H}_{78} \mathrm{O}_{19}$ & Chikusetsusaponin V methyl ester & Kondo et al. 1971 \\
\hline 89 & $\mathrm{~V}$ & $\begin{array}{c}\text { C.2, ACN, } 30-35 \%, 60 \mathrm{~min}, \\
35-40 \% 20 \mathrm{~min}\end{array}$ & $\mathrm{C}_{48} \mathrm{H}_{76} \mathrm{O}_{19}$ & Ginsenoside Ro & Matsuda et al. 1990 \\
\hline 90 & $\mathrm{~V}$ & $\begin{array}{l}\text { C.2, ACN, } 30-35 \%, 60 \mathrm{~min}, \\
35-40 \% 20 \mathrm{~min}\end{array}$ & $\mathrm{C}_{54} \mathrm{H}_{86} \mathrm{O}_{24}$ & Calendulaglycoside A & $\begin{array}{l}\text { Vidal-Ollivier et al. } \\
\qquad 1989\end{array}$ \\
\hline 91 & $\mathrm{~V}$ & $\begin{array}{c}\text { C. } 2, \mathrm{ACN}, 30-35 \%, 60 \mathrm{~min}, \\
35-40 \% 21 \mathrm{~min}\end{array}$ & $\mathrm{C}_{53} \mathrm{H}_{84} \mathrm{O}_{23}$ & Elatoside C & Yoshikawa et al. 1993 \\
\hline 92 & $\mathrm{~V}$ & $\begin{array}{l}24 \mathrm{~min}, \mathrm{C} .1, \mathrm{ACN}, 35-42 \% \\
80 \mathrm{~min}, 42-100 \% 5 \mathrm{~min}\end{array}$ & $\mathrm{C}_{47} \mathrm{H}_{74} \mathrm{O}_{18}$ & Pseudoginsenoside RT1 & Morita et al. \\
\hline 93 & $\mathrm{~V}$ & $\begin{array}{l}55 \mathrm{~min}, \mathrm{C} .1, \mathrm{ACN}, 35-42 \%, \\
80 \mathrm{~min}, 42-100 \% 5 \mathrm{~min}\end{array}$ & $\mathrm{C}_{43} \mathrm{H}_{68} \mathrm{O}_{14}$ & Silphioside A & Jiang et al. 1992 \\
\hline 94 & $\mathrm{~V}$ & $\begin{array}{l}46 \mathrm{~min}, \mathrm{C} .1, \mathrm{ACN}, 35-42 \% \\
80 \mathrm{~min}, 42-100 \% 5 \mathrm{~min}\end{array}$ & $\mathrm{C}_{48} \mathrm{H}_{76} \mathrm{O}_{18}$ & Umbellatoside B & Sosa et al. 2011 \\
\hline 95 & $\mathrm{~V}$ & $\begin{array}{c}20 \mathrm{~min}, \mathrm{C} .2, \mathrm{ACN}, 36 \%, 40 \\
\min \end{array}$ & $\mathrm{C}_{42} \mathrm{H}_{66} \mathrm{O}_{14}$ & Wedelin & Matos et al. 1983 \\
\hline 96 & $\mathrm{~V}$ & $\begin{array}{c}27.8 \mathrm{~min}, \mathrm{C} .2, \mathrm{ACN}, 57 \% \\
30 \mathrm{~min}, 57-62 \% \mathrm{~min} \\
62-70 \% 29 \mathrm{~min}\end{array}$ & $\mathrm{C}_{30} \mathrm{H}_{48} \mathrm{O}_{4}$ & 6ß-Hydroxyursolic acid & Sakakibara et al. 1983 \\
\hline 97 & $\mathrm{~V}$ & C. $2, \mathrm{ACN}, 35 \%$ & $\mathrm{C}_{30} \mathrm{H}_{48} \mathrm{O}_{6}$ & $\begin{array}{l}3 \beta, 6 \beta, 19 \alpha, 24-\text { tetrahydro xyurs-12-en-28-oic } \\
\text { acid }\end{array}$ & Fang et al. 1996 \\
\hline 98 & IV & $\begin{array}{c}71 \mathrm{~min}, \mathrm{C} .2, \mathrm{ACN}, 18 \% 110 \\
\min \end{array}$ & $\mathrm{C}_{36} \mathrm{H}_{58} \mathrm{O}_{11}$ & & Abe et al. 1987 \\
\hline 99 & V & $\begin{array}{l}38 \mathrm{~min}, \mathrm{C} .2, \mathrm{ACN}, 20-22 \% \\
75 \mathrm{~min}\end{array}$ & $\mathrm{C}_{36} \mathrm{H}_{58} \mathrm{O}_{11}$ & & Abe et al. 1987 \\
\hline 100 & $\mathrm{~V}$ & $\begin{array}{l}\text { 20min, C.2, ACN, } 36 \%, 40 \\
\text { min }\end{array}$ & $\mathrm{C}_{42} \mathrm{H}_{66} \mathrm{O}_{14}$ & Wedelin & Matos et al. 1983 \\
\hline 101 & IV & $\begin{array}{l}8 \mathrm{~min}, \mathrm{C} .2, \mathrm{MeOH}, 10-14 \% \\
40 \mathrm{~min}\end{array}$ & $\mathrm{C}_{37} \mathrm{H}_{55} \mathrm{~N}_{3} \mathrm{O}_{16}$ & Lycibarbarspermidine L & Zhou et al. 2016 \\
\hline 102 & IV & $\begin{array}{l}\text { C. } 2, \mathrm{MeOH}, 10-14 \%, 40 \\
\min \end{array}$ & $\mathrm{C}_{37} \mathrm{H}_{55} \mathrm{~N}_{3} \mathrm{O}_{16}$ & Lycibarbarspermidine $\mathrm{M}$ & Zhou et al. 2016 \\
\hline 103 & IV & $\begin{array}{l}\text { C. } 2, \mathrm{MeOH}, 10-14 \%, 40 \\
\min \end{array}$ & $\mathrm{C}_{37} \mathrm{H}_{53} \mathrm{~N}_{3} \mathrm{O}_{16}$ & Lycibarbarspermidine $\mathrm{E}$ & Zhou et al. 2016 \\
\hline 104 & IV & $\begin{array}{l}\text { C. } 2, \mathrm{MeOH}, 10-14 \%, 40 \\
\text { min }\end{array}$ & $\mathrm{C}_{37} \mathrm{H}_{53} \mathrm{~N}_{3} \mathrm{O}_{16}$ & & Jin et al. 2015 \\
\hline 105 & IV & $\begin{array}{l}\text { C. } 2, \mathrm{MeOH}, 10-14 \%, 40 \\
\min \end{array}$ & $\mathrm{C}_{31} \mathrm{H}_{43} \mathrm{~N}_{3} \mathrm{O}_{11}$ & Lycibarbarspermidine D & Zhou et al. 2016 \\
\hline
\end{tabular}


Table 2. Cont.

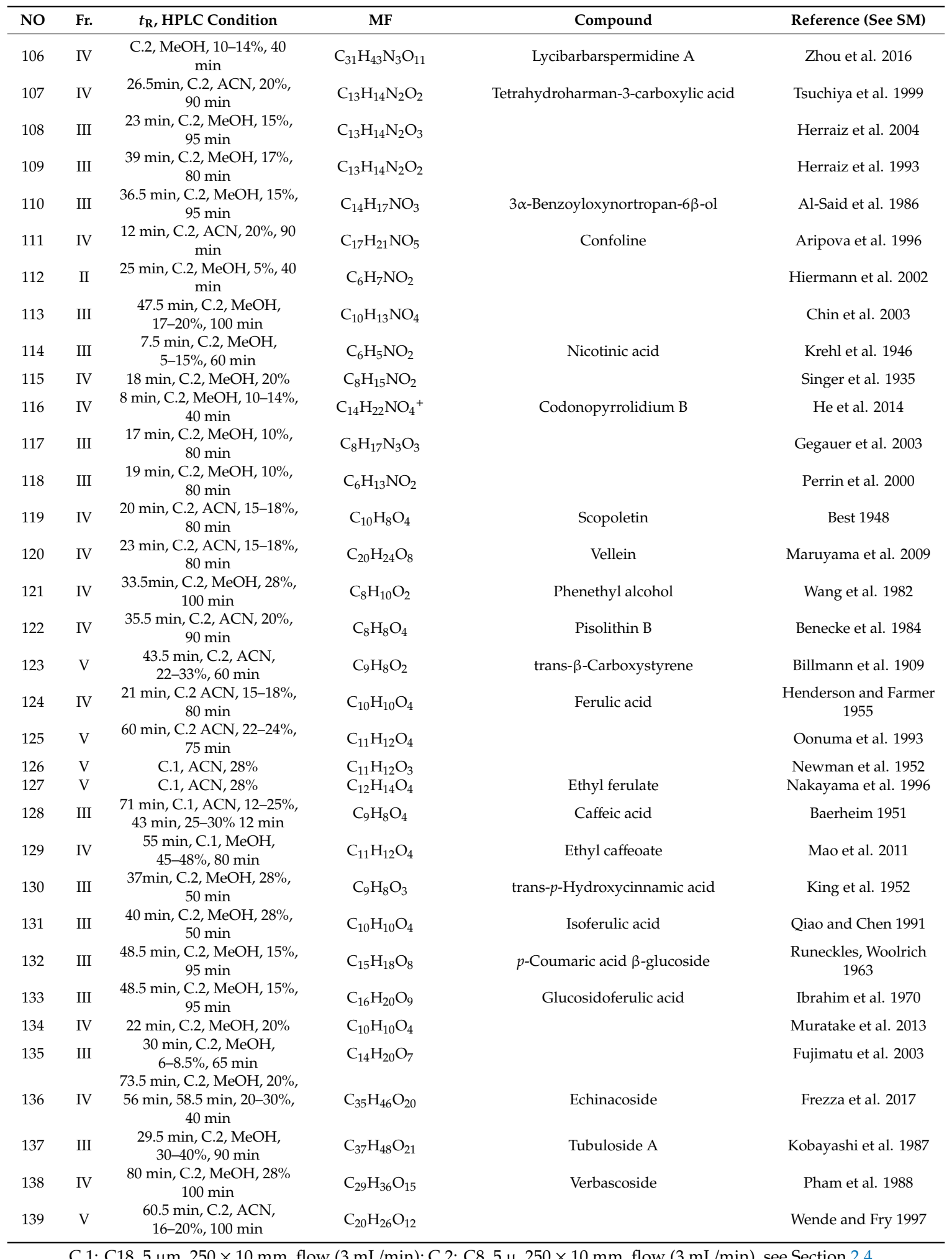


Table 3. Benzoquinone, naphthoquinone, anthraquinones, or phenanphrene derivatives (\#140-149), xanthones, chromone, and $\gamma$-pyrone derivatives (\#150-155), lignans (\#156-162), other aromatic compounds (\#163-175), and other types of compounds (\#176-189) isolated from Jing liqueur.

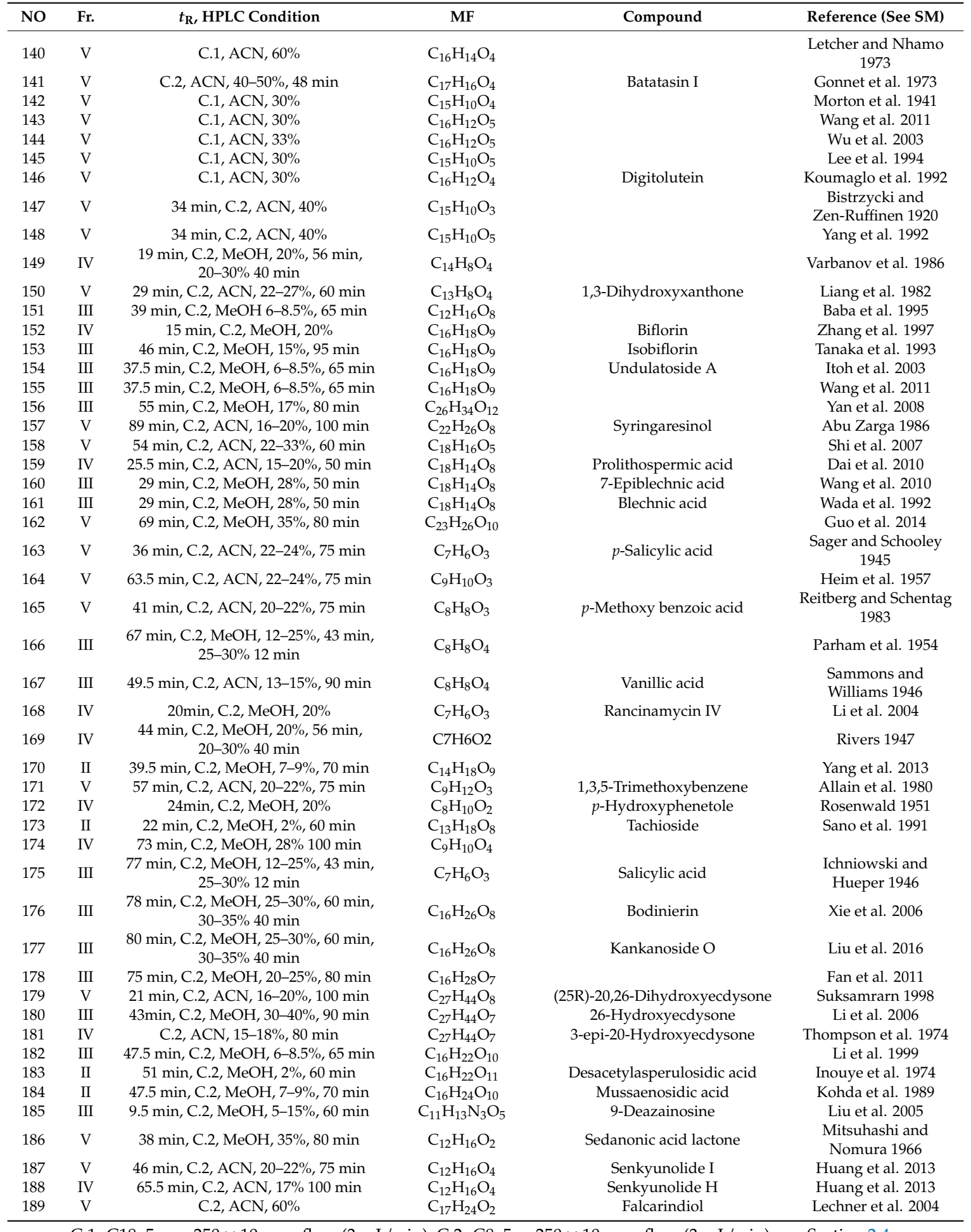

C.1: C18, $5 \mu \mathrm{m}, 250 \times 10 \mathrm{~mm}$, flow ( $3 \mathrm{~mL} / \mathrm{min})$; C.2: C8, $5 \mu, 250 \times 10 \mathrm{~mm}$, flow (3 mL/min), see Section 2.4 .

\subsection{Analysis of Metals, Amino Acids, and Total Polysaccharides}

Besides the isolation and structure determination of the above 189 compounds from Chinese herbal medicines, we determined the concentrations of two amino acids ( $L$-proline, $2.33 \mathrm{mg} / \mathrm{L}$; and 
L-arginine, $1.73 \mathrm{mg} / \mathrm{L}$ ), total amino acids $(9.84 \mathrm{mg} / \mathrm{L}$ ), and three metals (iron, $0.52 \mathrm{mg} / \mathrm{L}$; zinc, $0.21 \mathrm{mg} / \mathrm{L}$; and calcium, $11.0 \mathrm{mg} / \mathrm{L}$ ). The total amount of polysaccharides, the main component in fraction I was also determined $(337.4 \mathrm{mg} / \mathrm{L})$.

\subsection{Nrf2 Activation}

Results showed that a $5.2 \mathrm{mg} / \mathrm{mL}$ crude extract of Jing liqueur increased Nrf2 activity by approximately 7-8-fold (Figure 3). We also tested the five fractions from Jing liqueur. Nrf2 was strongly activated by fraction $V$ at $80 \mu \mathrm{g} / \mathrm{mL}$, weakly activated by fraction $\mathrm{V}$ and IV at 40 and $80 \mu \mathrm{g} / \mathrm{mL}$, respectively (Figure 4), indicating that fraction $\mathrm{V}$ contains most of the Nrf2 activators in Jing liqueur. Fraction V at $20 \mu \mathrm{g} / \mathrm{mL}$, fraction IV at $40 \mu \mathrm{g} / \mathrm{mL}$, and both fractions III and II at $80 \mu \mathrm{g} / \mathrm{mL}$ marginally activated Nrf2, while fraction I was inactive. Next, we screened almost all the 189 isolated molecules except twenty one (21) because of their insufficiency. Eighteen (18) compounds showed Nrf2 activation when compared with the negative control (Figure 5). Among these eighteen Nrf2 activators (Figures 1 , 2 and 5), four $(55,78, \mathbf{1 2 9}$, and 168) from fraction IV, and the other fourteen $(\mathbf{5 0}, \mathbf{5 1}, \mathbf{5 3}, \mathbf{5 8}, \mathbf{1 2 5}, \mathbf{1 2 6}$, $127,128,143,144,145,146,171$, and 186) from fraction V (Table 4). Compounds 55, 78, 129, and 168 from fraction IV and 50, 53, 58, 128, 143, 144, 145, 146, 171, and 186 from fraction V were weakly active. When comparing the activity of the active compounds from fraction $V$, compounds 51, 125, 126, and 127 showed much stronger activity than the other Nrf2 activators, which robustly enhanced the Nrf2 expression at $40 \mu \mathrm{g} / \mathrm{mL}$ (Table 4 ).

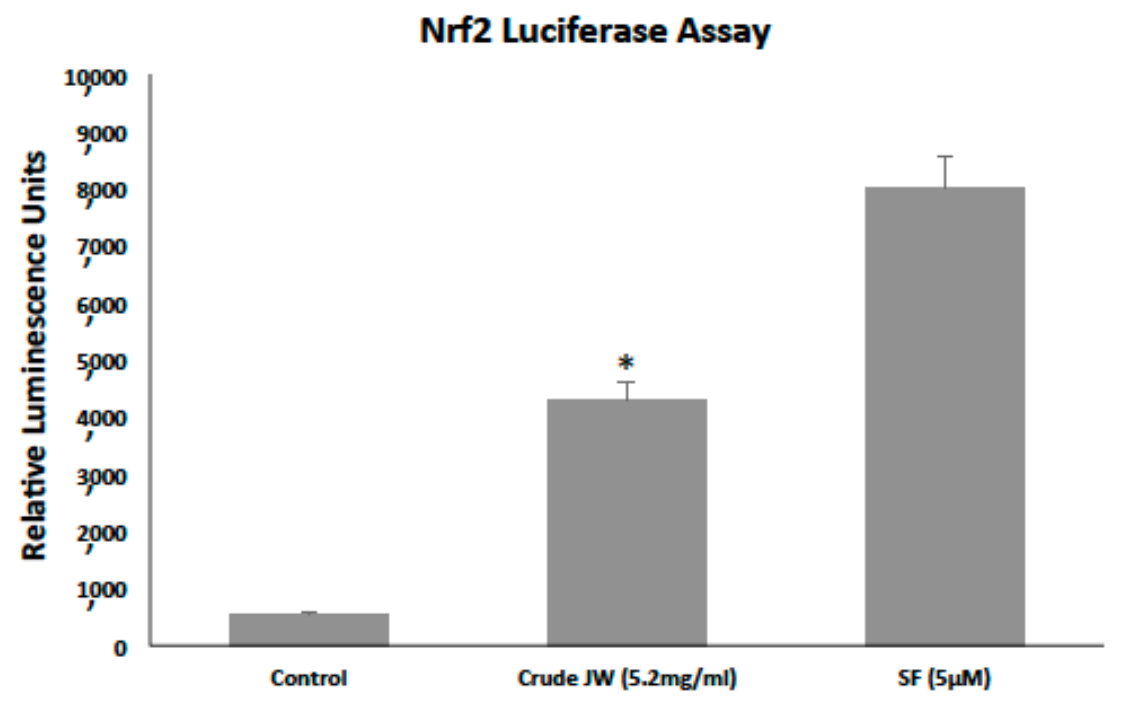

Figure 3. The effect of the crude on Nrf2 in ARE reporter-Hep G2 cells. Cells were seeded in 96-well plates at a density of $4 \times 10^{4}$ cells/well and incubated for $24 \mathrm{~h}$. The cells were further treated with $5.2 \mathrm{mg} / \mathrm{mL}$ concentration of crude Jing liqueur for additional $24 \mathrm{~h}$. The negative control cells were treated with $0.2 \%$ DMSO (dimethyl sulfoxide), and positive control cells were treated with $5 \mu \mathrm{M}$ DL-sulforaphane (SF). Luciferase activity was determined. ${ }^{*} p<0.05$. 


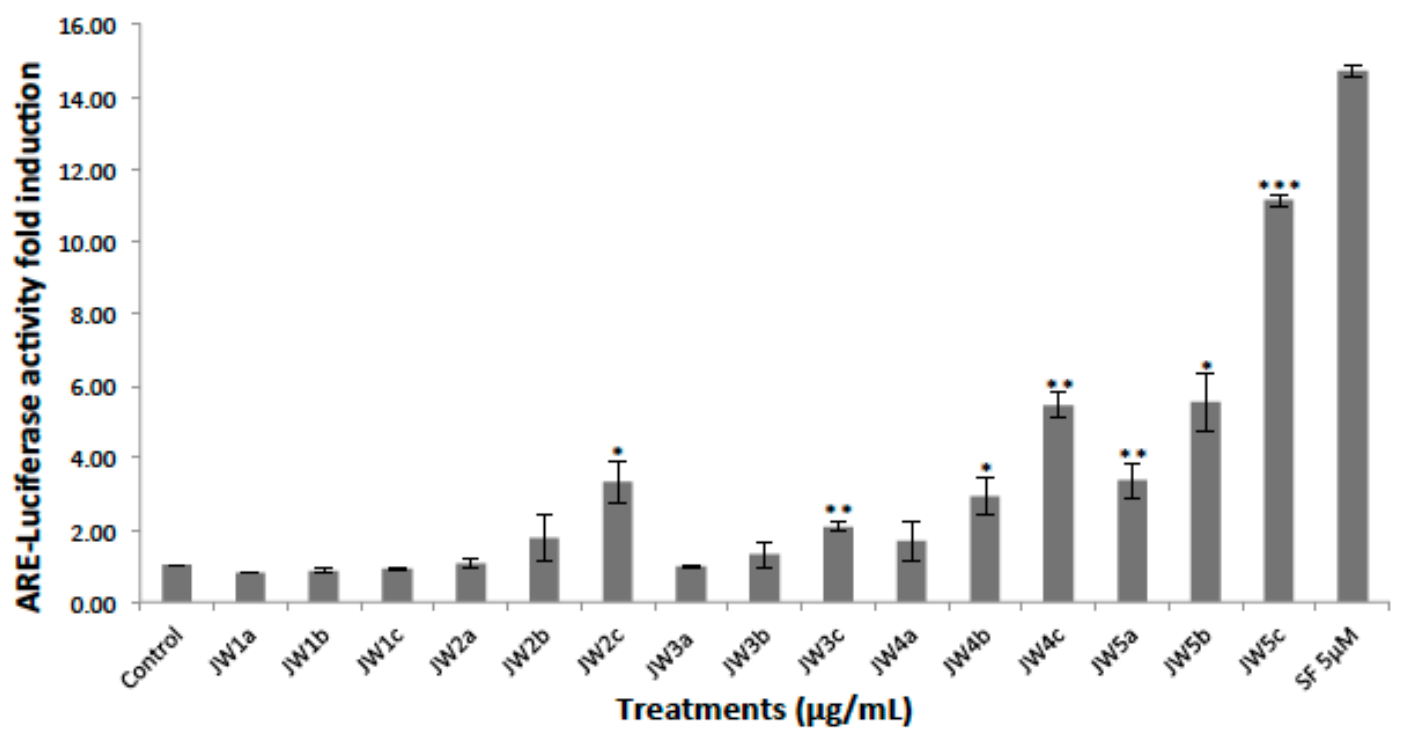

Figure 4. The effect of five fractions on ARE-luciferase reporter activity in ARE reporter-HepG2 cells. Cells were seeded in 96-well plates at a density of $4 \times 10^{4}$ cells/well and incubated for $24 \mathrm{~h}$. The cells were further treated with $20,40,80 \mu \mathrm{g} / \mathrm{mL}$ concentrations of each fraction for additional $24 \mathrm{~h}$. The negative control cells were treated with $0.2 \%$ DMSO, and positive control cells were treated with $5 \mu \mathrm{M}$ DL-sulforaphane (SF). Luciferase activity was determined. Mean ARE-luciferase reporter activity represents the average of three-independent experiments \pm S.E.M. ${ }^{*} p<0.05 ;{ }^{* *} p<0.01,{ }^{* * *} p<0.001$. (a: $20 \mu \mathrm{g} / \mathrm{mL}$; b: $40 \mu \mathrm{g} / \mathrm{mL}$; : $80 \mu \mathrm{g} / \mathrm{mL}$ ).

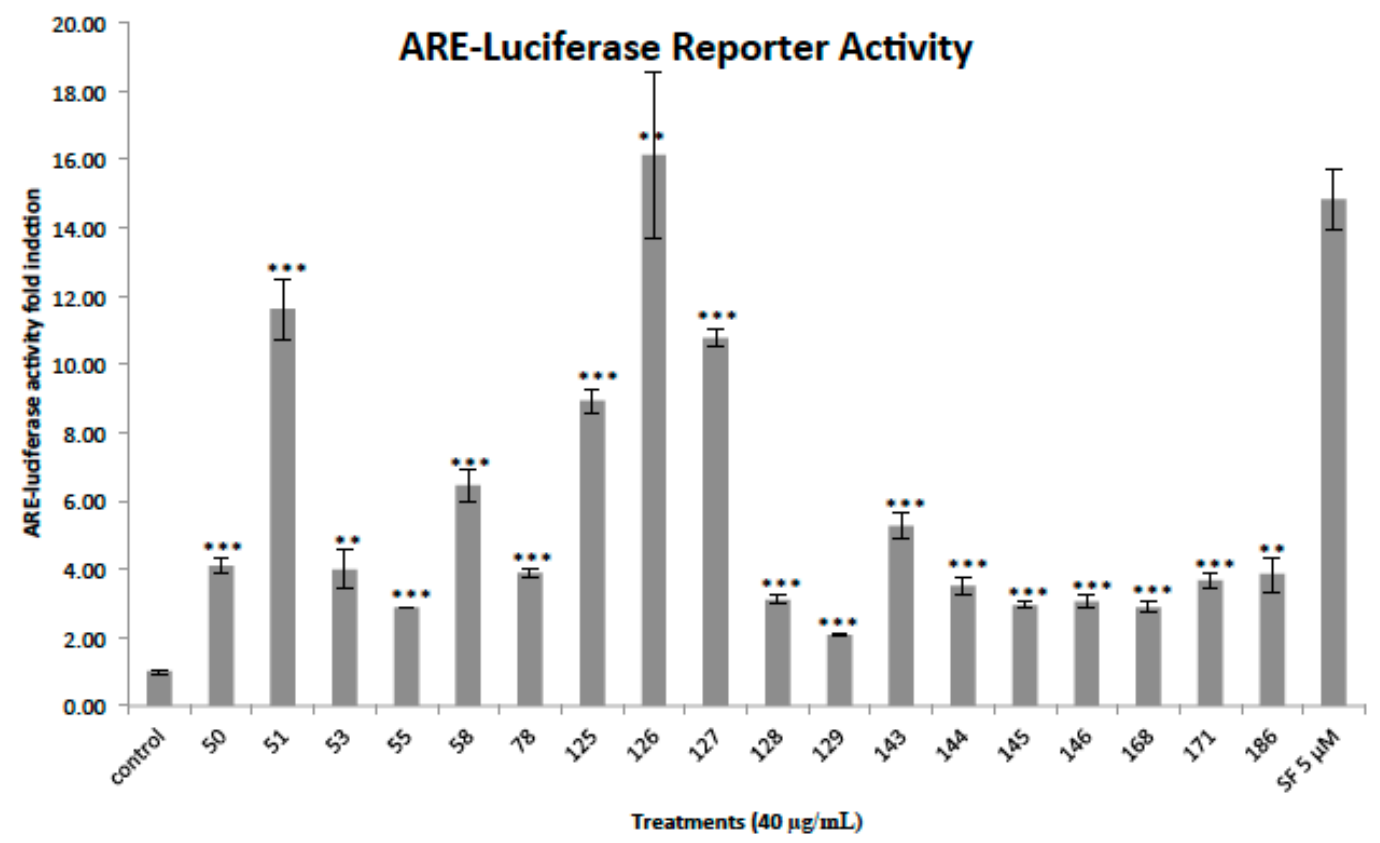

Figure 5. The effect of pure compounds on ARE-luciferase reporter activity in ARE repoter-HepG2 cells. Cells were seeded in 96-well plates at a density of $4 \times 10^{4}$ cells/well and incubated for $24 \mathrm{~h}$. The cells were further treated with compounds $(40 \mu \mathrm{g} / \mathrm{mL}$ each) for additional $24 \mathrm{~h}$. The negative control cells were treated with $0.2 \%$ DMSO, and positive control cells were treated with $5 \mu \mathrm{M}$ DL-sulforaphane (SF). Luciferase activity were determined. Mean ARE-luciferase reporter activity represents the average of three-independent experiments \pm S.E.M. ${ }^{* *} p<0.01,{ }^{* * *} p<0.001$. 
Table 4. Relative Nrf2 activity induced by compounds $(40 \mu \mathrm{g} / \mathrm{mL})$ isolated from Jing liqueur.

\begin{tabular}{ccccccccc}
\hline Cpd & Fr. & $\begin{array}{c}\text { Relative Nrf2 } \\
\text { Activity (Fold } \\
\text { Induction) }\end{array}$ & Cpd & Fr. & $\begin{array}{c}\text { Relative Nrf2 } \\
\text { Activity (Fold } \\
\text { Induction) }\end{array}$ & Cpd & Fr. & $\begin{array}{c}\text { Relative Nrf2 } \\
\text { Activity (Fold } \\
\text { Induction) }\end{array}$ \\
\hline Control & - & 1.0 & $\mathbf{1 2 5}$ & $\mathrm{V}$ & 9.5 & $\mathbf{1 4 5}$ & $\mathrm{V}$ & 3.0 \\
$\mathbf{5 0}$ & $\mathrm{V}$ & 4.0 & $\mathbf{1 2 6}$ & $\mathrm{V}$ & 16.0 & $\mathbf{1 4 6}$ & $\mathrm{V}$ & 3.0 \\
$\mathbf{5 1}$ & $\mathrm{V}$ & 11.5 & $\mathbf{1 2 7}$ & $\mathrm{V}$ & 10.5 & $\mathbf{1 6 8}$ & $\mathrm{IV}$ & 3.0 \\
$\mathbf{5 3}$ & $\mathrm{V}$ & 4.0 & $\mathbf{1 2 8}$ & $\mathrm{V}$ & 3.0 & $\mathbf{1 7 1}$ & $\mathrm{V}$ & 3.5 \\
$\mathbf{5 5}$ & $\mathrm{IV}$ & 3.0 & $\mathbf{1 2 9}$ & $\mathrm{IV}$ & 2.2 & $\mathbf{1 8 6}$ & $\mathrm{V}$ & 3.5 \\
$\mathbf{5 8}$ & $\mathrm{V}$ & 6.5 & $\mathbf{1 4 3}$ & $\mathrm{V}$ & 6.0 & $\mathrm{SF}(5 \mu \mathrm{M})$ & - & 15.0 \\
$\mathbf{7 8}$ & $\mathrm{IV}$ & 4.0 & $\mathbf{1 4 4}$ & $\mathrm{V}$ & 3.5 & & & \\
\hline
\end{tabular}

\subsection{Cytotoxicity Evaluation}

In order to evaluate the cytotoxicity of Jing liqueur, we used the MTT assay to measure the activity of the crude and the five fractions. We tested the five fractions (I-V) at 20,40, and $80 \mu \mathrm{g} / \mathrm{mL}$, and none exhibited cytotoxicity as shown by the MTT results (Figure 6). The Nrf2 activators identified in this study were also evaluated for the activity against HepG2 in our MTT assay, and none of them showed any cytotoxicity at $40 \mu \mathrm{g} / \mathrm{mL}$.

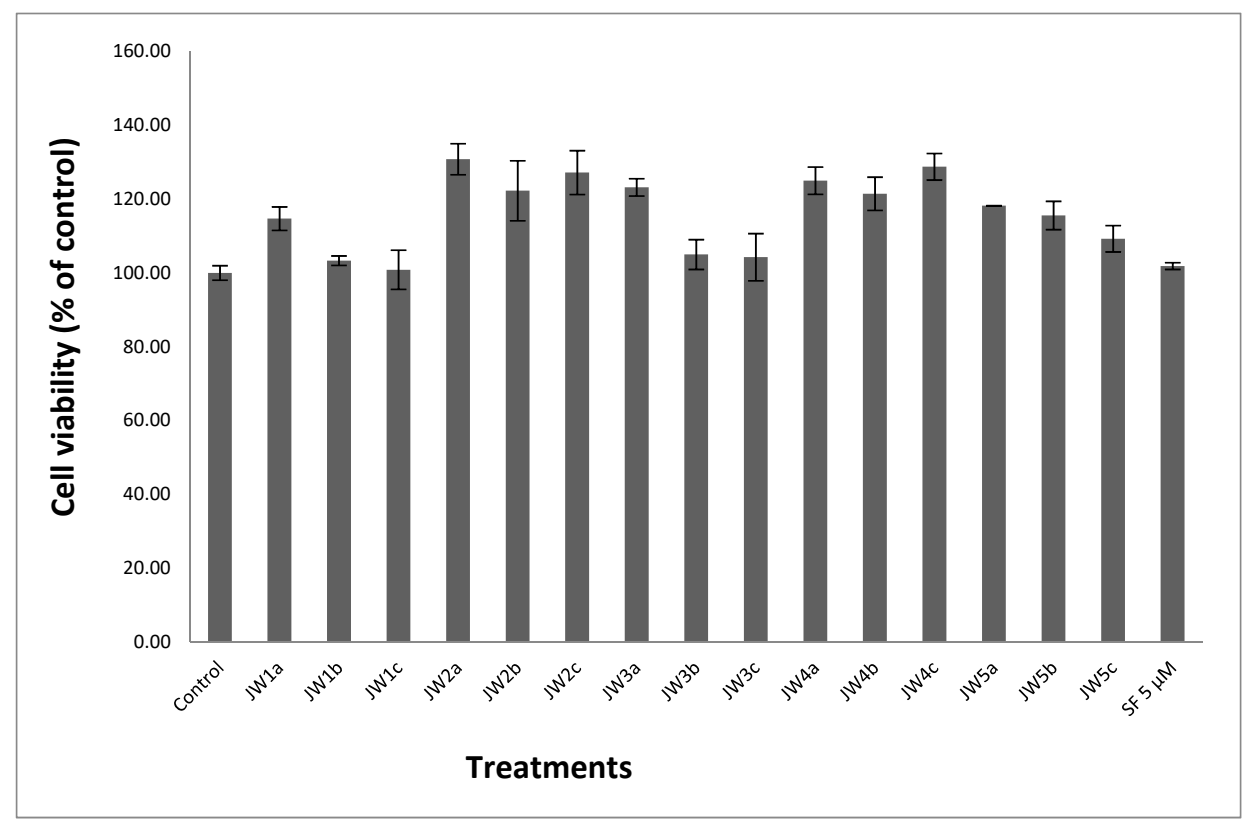

Figure 6. Evaluation of cytotoxicity activity of five fractions in ARE reporter-HepG2 cells. Cells were seeded in 96-well plates at a density of $4 \times 10^{4}$ cells/well and incubated for $24 \mathrm{~h}$. The cells were further treated with $20,40,80 \mu \mathrm{g} / \mathrm{mL}$ concentrations of each fraction for additional $24 \mathrm{~h}$. The negative control cells were treated with $0.2 \%$ DMSO cells. Cell viability was estimated with the methylthiazoltetrazolium (MTT) assay. Mean cytotoxicity activity represents the average of three-independent experiments \pm S.E.M. (a: $20 \mu \mathrm{g} / \mathrm{mL}$; b: $40 \mu \mathrm{g} / \mathrm{mL}$; c: $80 \mu \mathrm{g} / \mathrm{mL})$.

\section{Discussion}

One hundred eighty nine compounds have been isolated from Jing liqueur. Most of them are aromatic compounds including 78 flavonoids, 21 coumarins, cinnamic acid, or coumaric acid, and phenyl ethanol (or acetic acid) derivatives, 10 benzoquinone, naphthoquinone, anthraquinones, or phenanphrene derivatives, 6 xanthones, chromone, and $\gamma$-pyrone derivatives, 7 lignans, and 13 small aromatic compounds. The three major types of compounds are flavonoids, terpenoids, and alkaloids, and they have a broad range of biological activities including anti-oxidant, anti-inflammatory, antibacterial, and anticancer properties etc. These aromatic compounds, especially flavonoids, 
anthraquinones, cinnamic acid derivatives, lignans, and some other small molecule aromatic compounds, are probably the main anti-oxidant components in Jing liqueur.

$L$-proline and $L$-arginine are two of the six conditionally essential amino acids [21,22]. These amino acids and elements are important for heart muscle, immune function, blood production, blood pressure regulation, and prevention of osteoporosis etc. Iron is an essential element for blood production [23]. Zinc is extremely important for the body's defense (immune) system to work properly, and plays a role in cell division, cell growth, wound healing, and the breakdown of carbohydrates [24]. Calcium helps to form and maintain healthy teeth and bones, which is important for the prevention of osteoporosis $[25,26]$. Olysaccharides are the most abundant type of compounds in Jing liqueur. We have a good reason to argue that olysaccharides together with flavonoids and terpenoids may account for some other biological activities besides the anti-oxidant property of Jing liqueur.

Fractions II-V were active in the Nrf2 assay at $80 \mu \mathrm{g} / \mathrm{mL}$, fraction $\mathrm{V}$ was much more active than fractions II-IV, and fraction IV was more active than fractions II and III. Since most of the 189 compounds were isolated from fractions IV and V, majority of which are flavonoids, cinnamic acid derivatives, lignans, and other aromatic compounds, we argued that it is likely that Nrf2 activators in Jing liqueur are aromatic molecules. We evaluated the 168 compounds that were enough for the screening, and eighteen compounds activated Nrf2. Among these eighteen Nrf2 activators four were isolated from fraction IV, and the other fourteen were separated from fraction V. Clearly, most of the active compounds and the four most active Nrf2 activators (51 and 125-127) identified in this study were isolated from fraction $\mathrm{V}$, which was consistent with our screening result of the five fractions with fraction $\mathrm{V}$ being the most active fraction. Almost all these eighteen Nrf2 activators are aromatic molecules except 186, including six flavonoids (50, 51, 53, 55, 58, and 78), five cinnamic acid derivatives (125-129), four anthraquinones (143-146), 3,4-dihydroxybenzaldehyde (168), 1,3,5-trimethoxybenzene (171), and (E)-3-butylidene-4,5,6,7-tetrahydroisobenzofuran-1(3H)-one (186). Jing liqueur, the five fractions at 80 $\mu \mathrm{g} / \mathrm{mL}$ and the eighteen Nrf2 activators at $40 \mu \mathrm{g} / \mathrm{mL}$ were evaluated for their antiproliferative activity against HepG2, and none showed any cytotoxicity.

This study is significant because it was the first time to extensively investigate Jing liqueur chemically that has not been previously interrogated although the phytochemical components and biological activities of these nine Chinese plants as a single herbal medicine have been investigated. The Nrf2 activators especially compounds 51, 125, 126, and 127 identified in this study could be used as biomarkers for quality control. Jing liqueur was reported to exhibit anti-inflammatory [1], immune enhancement [3], anti-fatigue [2,3] properties, and invigorating the vital activities of kidney [3]. Our study showed that the Nrf2 activation by Jing liqueur may account for the observed anti-inflammatory activity and immune enhancement of Jing liqueur. These experiments also demonstrated that to drink certain volume of Jing liqueur equivalent to the highest concentration tested in these experiments should be safe regarding the cytotoxicity of the metabolites of the herbal medicine in Jing liqueur. Hence, adequate consumption of Jing liqueur may offer health benefits mainly or partially because of the transient activation of Nrf2 considerably by the above-mentioned eighteen Nrf2 activators present in Jing liqueur. Of course, excessive drinking is not encouraged.

\section{Conclusions}

We isolated 189 compounds from fractions II-V of Jing liqueur, one of which (7) was a minor new flavonoid. Out of these 189 compounds, 78 are flavonoids, revealing the Jing liqueur is rich in phenolic compounds. The concentrations of most compounds were at micromolar levels (corresponding to $\mu \mathrm{g} / \mathrm{L}$ levels). Fraction I was mainly composed of polysaccharides. The concentration of total polysaccharides was very high $(337.4 \mathrm{mg} / \mathrm{L})$, which may be worthy of further study for the components and functions. Both iron and zinc were less than $1 \mathrm{mg} / \mathrm{L}$ while the concentration of calcium was much higher (11 $\mathrm{mg} / \mathrm{L}$ ). $L$-proline and $L$-arginine were at $\mathrm{mg} / \mathrm{L}$ levels. We also demonstrated that the crude extract of Jing liqueur, fractions II-V activated the Nrf2 transcription factor pathway, and fraction V was much more active than fractions II-IV, indicating that fraction V contains more Nrf2 activators than 
fractions II-IV. Screening of compounds demonstrated that most (14) of the eighteen active compounds including the two most potent Nrf2 activators (51 and 126) were isolated from fraction V. Nrf2 is an important defense mechanism for mitigating oxidative and electrophilic stress. Despite the "dark side" [27], Nrf2 activation is believed to have many beneficial effects on human health including inhibition of systemic inflammation, cancer prevention, relief of diabetes-induced cardiac oxidative stress, and neuroprotection. The activation of Nrf2 is highly consistent with the traditional use of the herbal medicines present in Jing liqueur, which itself is known for its tonic effects including general health and well-being promotion. Many of the reported beneficial properties of Jing liqueur including anti-inflammatory [1], immune enhancement [2], and anti-fatigue [2,3] properties could at least partially be justified by the presence of different types of compounds including Nrf2 activators. The crude extract and the five fractions were not cytotoxic against HepG2 cells at $80 \mu \mathrm{g} / \mathrm{mL}$, and the compounds that activated Nrf2 were also not active in our MTT cytotoxicity assay at $40 \mu \mathrm{g} / \mathrm{mL}$, the highest concentration of compounds tested in the Nrf2 assay. Further investigation of Jing liqueur on the Nrf2 pathway is warranted.

Supplementary Materials: The following are available online at http://www.mdpi.com/2306-5710/6/1/1/s1. Figure S1: Structure of 7. Table S1: ${ }^{1} \mathrm{H}$ and ${ }^{13} \mathrm{C}$ NMR data of 7 (400 MHz, CD $\mathrm{CD}_{3}$ ). Figure S2: HR-ESI-MS spectrum of 7. Figure S3: ${ }^{1} \mathrm{H}-\mathrm{NMR}$ spectrum of 7 (400 MHz, CD $\mathrm{CD}_{3} \mathrm{OD}$ ). Figure S4: COSY (Correlation Spectroscopy) spectrum of 7 (400 MHz, CD 3 OD). Figure S5: HSQC (Heteronuclear Single Quantum Coherence) spectrum of $7\left(400 \mathrm{MHz}, \mathrm{CD}_{3} \mathrm{OD}\right)$. Figure S6: HMBC (Heteronuclear Multiple Bond Correlation) spectrum of $7(400 \mathrm{MHz}$, $\left.\mathrm{CD}_{3} \mathrm{OD}\right)$. MS, NMR, and references of the other known compounds.

Author Contributions: Y.-S.C., J.X., M.C., Y.L. and S.C. designed research; Y.-S.C., J.X., M.C., Y.Y., A.M., D.W. and X.W. performed research; Y.-S.C. and S.C. analyzed data; and Y.-S.C. and S.C. wrote the paper. All authors have read and agreed to the published version of the manuscript.

Funding: The authors are grateful to Jing Brand Research Institute, Jing Brand Co., Ltd. for financial support.

Acknowledgments: We thank Aaron Jacobs for his advice and help in the Nrf2 assay and manuscript preparation.

Conflicts of Interest: The authors declare no conflict of interest.

\section{Abbreviations}

Traditional Chinese medicine (TCM); Chinese Materia Medica (CMM); nuclear factor erythroid 2-related factor 2 (Nrf2); antioxidant responsive element (ARE); high performance liquid chromatography (HPLC); nuclear magnetic resonance (NMR); liquid chromatography-mass spectrometry (LC-MS); methylthiazoltetrazolium (MTT).

\section{References}

1. Feng, S.; Shan, Y.; Lu, S.; Liu, Y.; He, G. The anti-inflammatory effect of moderate drinking. Liquor-Mak. Sci. Tech. 2013, 229, 121-124.

2. Lu, S.; Feng, S.; Li, J.; Yin, T.; Shi, Y.; Shi, J.; Chen, Y.; Liu, Y. Fatigue mitigating effect of Chinese Jing liqueur in sub-health patients. West. J. Trad. Chi. Med. 2017, 30, 82-84.

3. Shan, Y.; Zhou, H.; Chen, M.; Chen, K.; Liu, Y.; Wang, L. Study on anti-fatigue, regulating immunity and enhancing sexual function of Chinese Jing liqueur. Trad. Chin. Pat. Med. 2018, 40, 1600-1603.

4. Liu, J.; Zhao, Z.Z.; Chen, H.B. Review of astragali radix. Chin. Herb. Med. 2011, 3, 90-105.

5. Wang, L.L.; Ding, H.; Yu, H.S.; Han, L.F.; Lai, Q.H.; Zhang, L.J.; Song, X.B. Cistanches herba: Chemical constituents and pharmacological effects. Chin. Herb. Med. 2015, 7, 135-142. [CrossRef]

6. Li, Z.Q.; Cao, W.F. Research progress in Dioscorea opposita and major active components quick view other sources. Chin. J. Gerontol. 2013, 33, 1975-1976.

7. Potterat, O. Goji (Lycium barbarum and L. chinense): Phytochemistry, pharmacology and safety in the perspective of traditional uses and recent popularity. Plant. Med. 2010, 76, 7-19. [CrossRef]

8. Chen, X.J.; Tang, Z.H.; Li, X.W.; Xie, C.X.; Lu, J.J.; Wang, Y.T. Chemical constituents, quality control, and bioactivity of Epimedii folium (Yinyanghuo). Am. J. Chin. Med. 2015, 43, 785-834. [CrossRef]

9. Luo, Q.; Wang, S.M.; Lu, Q.; Luo, J.; Cheng, Y.X. Identification of compounds from the water soluble extract of Cinnamomum cassia barks and their inhibitory effects against high-glucose-induced mesangial cells. Molecules 2013, 18, 10930-10943. [CrossRef] 
10. Mittal, M.; Gupta, N.; Parashar, P.; Mehra, V.; Khatri, M. Phytochemical evaluation and pharmacological activity of syzygium aromaticum: A comprehensive review. Int. J. Pharm. Pharm. Sci. 2014, 18, 10930-10943.

11. Wei, W.L.; Zeng, R.; Gu, C.M.; Qu, Y.; Huang, L.F. Angelica sinensis in China-A review of botanical profile, ethnopharmacology, phytochemistry and chemical analysis. Int. J. Ethnopharmacol. 2016, 190, 116-141. [CrossRef] [PubMed]

12. Liu, X.; Zhang, B.; Chou, G.; Yang, L.; Wang, Z. Chemical constituents from imperata cylindrical. China J. Chin. Mat. Med. 2012, 37, 2296-2300.

13. Zhang, M.; An, C.; Gao, Y.; Rehana, K.; Leak, R.K.; Chen, J.; Zhang, F. Emerging roles of Nrf2 and phase II antioxidant enzymes in neuroprotection. Prog. Neurobiol. 2013, 100, 30-47. [CrossRef] [PubMed]

14. Sporn, M.B.; Liby, K.T. NRF2 and cancer: The good, the bad and the importance of context. Nat. Rev. Cancer 2012, 12, 564-571. [CrossRef]

15. Thimmulappa, R.K.; Lee, H.; Rangasamy, T.; Reddy, S.P.; Yamamoto, M.; Kensler, T.W.; Biswal, S. Nrf2 is a critical regulator of the innate immune response and survival during experimental sepsis. J. Clin. Investig. 2006, 116, 984-995. [CrossRef]

16. Matzinger, M.; Fischhuber, K.; Heiss, E.H. Activation of Nrf2 signaling by natural products-can it alleviate diabetes? Biotechnol. Adv. 2018, 36, 1738-1767. [CrossRef]

17. Rojo de la Vega, M.; Chapman, E.; Zhang, D.D. NRF2 and the Hallmarks of Cancer. Cancer Cell 2018, 34, 21-43. [CrossRef]

18. Guo, N.; Bai, Z.; Jia, W.; Sun, J.; Wang, W.; Chen, S.; Wang, H. Quantitative Analysis of Polysaccharide Composition in Polyporus umbellatus by HPLC-ESI-TOF-MS. Molecules 2019, 24, 2526. [CrossRef]

19. Li, W.-K.; Xiao, P.-G.; Liao, M.-C.; \& Zhang, R.-Y. Caohuoside-C from the aerial parts of Epimedium koreanum Nakai. Gaodeng Xuexiao Huaxue Xuebao 1995, 16, 230-233.

20. Kanwal, N.; Siddiqui, A.J.; Haq, F.U.; El-Seedi, H.R.; Musharraf, S.G. Two-stage mass spectrometry approach for the analysis of triterpenoid glycosides in Fagonia indica. RSC Adv. 2018, 8, 41023-41031. [CrossRef]

21. Wu, G.; Bazer, F.W.; Burghardt, R.C.; Johnson, G.A.; Kim, S.W.; Knabe, D.A.; Li, P.; Li, X.; McKnight, J.R.; Satterfield, M.C.; et al. Proline and hydroxyproline metabolism: Implications for animal and human nutrition. Amino Acids 2011, 40, 1053-1063. [CrossRef] [PubMed]

22. Chin-Dusting, J.; Alexander, C.; Arnold, P.; Hodgson, W.; Lux, A.; Jennings, G. Effects of In Vivo and In Vitro L-Arginine Supplementation on Healthy Human Vessels. J. Cardiovasc. Pharm. 1996, 28, 158-166. [CrossRef] [PubMed]

23. Abbaspour, N.; Hurrell, R.; Kelishadi, R. Review on iron and its importance for human health. J. Res. Med. Sci. 2014, 19, 164-174. [PubMed]

24. Prasad, A.S. Zinc in Human Health: Effect of Zinc on Immune Cells. Mol. Med. 2008, 14, 353-357. [CrossRef] [PubMed]

25. Beto, J.A. The Role of Calcium in Human Aging. Clin. Nutr. Res. 2015, 4, 1-8. [CrossRef]

26. Cashman, K.D. Calcium intake, calcium bioavailability and bone health. Br. J. Nutr. 2002, 87 (Suppl. 2), S169-S177. [CrossRef]

27. Grossman, R.; Ram, Z. The dark side of Nrf2. World Neurosurg. 2013, 80, 284-286. [CrossRef]

(C) 2019 by the authors. Licensee MDPI, Basel, Switzerland. This article is an open access article distributed under the terms and conditions of the Creative Commons Attribution (CC BY) license (http://creativecommons.org/licenses/by/4.0/). 